\title{
Gaussian Recursive Filter for Nonlinear Systems with Finite-step Correlated Noises and Packet Dropout Compensations
}

\author{
Li-Guo Tan ${ }^{1}$, Cheng $\mathrm{Xu}^{2}{ }^{2}$, Yu-Fei Wang ${ }^{3}$, Hao-Nan Wei ${ }^{3}$, Kai Zhao ${ }^{4, *}$, Shen-Min Song ${ }^{4}$ \\ ${ }^{1}$ Research Center of Basic Space Science, Harbin Institute of Technology, Harbin 150001, China \\ ${ }^{2}$ Science and Technology on Complex System Control and Intelligent Agent Cooperation Laboratory, Beijing 100074, China \\ ${ }^{3}$ Beijing Electro-mechanical Engineering Institute, Beijing 100074, China \\ ${ }^{4}$ Control Science and Engineering, Harbin Institute of Technology, Harbin 150001, China \\ *Corresponding author: xch2000_1980@163.com,1014553342@qq.com
}

\begin{abstract}
This paper is focused on the nonlinear state estimation problem with finite-step correlated noises and packet loss. Firstly, by using the projection theorem repeatedly, the mean and covariance of process noise and measurement noise in the condition of measurements before the current epoch are calculated. Then, based on the Gaussian approximation recursive filter (GASF) and the prediction compensation mechanism, one-step predictor and filter with packet dropouts are derived, respectively. Based on these, a nonlinear Gaussian recursive filter is proposed. Subsequently, the numerical implementation is derived based on the cubature Kalman filter (CKF), which is suitable for general nonlinear system and with higher accuracy compared to the algorithm expanded from linear system to nonlinear system through Taylor series expansion. Finally, the strong nonlinearity model is used to show the superiority of the proposed algorithm.
\end{abstract}

Keywords: Gaussian recursive filter, dropout compensations, Gaussian approximation, numerical implementation.

\section{INTRODUCTION}

In the past few years, networked control systems (NCSs) have caused widespread concern [1]-[3], because they link the cyberspace and physical space, including networks among sensors, estimators, controller, and actuators. However, as NCSs become more and more complex, various non-ideal situations arise in the system model, such as nonlinearity, correlated noises, packet loss and so on.

For a nonlinear system, two types of methods are developed. One is based on approximating a nonlinear system, while another is based on approximating the probability distribution. For the former, the representative methods are the extended Kalman filter (EKF) [4] and the divided difference filter (DDF) [5]. For the latter, there are the Gauss-Hermite quadrature filter (GHQF) based on the Gauss-Hermite quadrature rule [6], the unscented Kalman filter (UKF) based on the unscented transformation [5], the CKF based on the spherical-radial cubature rule [7], and particle filter (PF) based on random sampling [8]. Apparently, CKF has a smaller computational burden than GHQF and PF, has higher precision than EKF, and has higher numerical stability than UKF.

For the systems with correlated noises, methods can be summarized into three categories, including decoupled framework based on the reconstruction of the pseudo-process equation, which makes the process noise uncorrelated with measurement noise [9]; GASF, based on a novel two-step prediction method, which avoids calculating the mean and covariance of process noise in the condition of the measurement at the same epoch [10]; alternative framework based on the state augmented by process noise and the conditional Gaussian distribution [11]. In [11], it proves that the algorithms in [9], [10], and [11] are equivalent in linear systems. In [12] and [13], two alternative frameworks proposed in [11] are modified and used to design nonlinear Gaussian filtering algorithms, respectively. In [14] and [15], a more complex form of noise is considered in linear and nonlinear systems, respectively. In [16] and [17], finite-step correlated noise is considered for optimal linear estimators and distributed fusion filter, respectively. It can be known from [16] that finite-step correlation noise is a more general case.

For the problem of packet dropouts, three different compensation mechanisms are proposed, respectively. In [18] and [19], based on the zero-input compensation mechanism, two different models are designed and corresponding estimators are proposed for data packets arriving at the filter within and without a sampling interval. To find an alternative measurement, the hold-input compensation mechanism is proposed. In [20], a white binary distributed random variable 
is used to describe that the sensor measurement received by the data processing center is from the current or last epoch. In [21], due to the communication constraint, only one network node is allowed to gain access to a shared communication channel and the optimal weighting factor is introduced to describe this situation. However, the optimal weighting factor is not shown in [21], and an improved version of the compensation mechanism is given in [22], where the weighting factor is replaced by a matrix, and the simulation results show the superiority of [22] compared with [21]. For the reason that the latest information on measurements is not used in hold-input compensation, the prediction compensation mechanism is proposed and shows a better filtering effect. In [23], the predictor of a lost packet is used. In [16] and [24], based on the same compensation strategy, delayed measurements, finite-step correlated noises and packet loss are considered, respectively. In [25], fusion algorithms for systems with multi-sensor measurement are considered in which different sensors have different packet loss rates.

Since finite-step correlated noise is widely present in practical systems, and packet dropout is an inherent problem in NCSs, it is very necessary to design estimation methods for systems with finite-step correlated noises and packet loss. However, for such problems, there is no unified framework for nonlinear system in existing literatures. In this paper, considering finite-step correlated noises and packet loss for general nonlinear systems under the Gaussian framework, we derive the nonlinear Gaussian recursive filter. It is worth mentioning that other form correlated noises or the system without packet loss is a special case of this article.

The rest of this paper is organized in the following order: The problem formulation is shown in Section 2. The main results are derived in Section 3. The numerical implementation is presented in Section 4. Section 5 gives the simulation result. The conclusion is shown in Section 6 .

Notation: $|\eta|$ denotes the absolute value of $\eta \cdot \boldsymbol{x}_{k \mid k-n}$ represents the mathematical expectation of $\boldsymbol{x}_{k}$ in the condition of $\boldsymbol{Y}_{k-n}$, where $\boldsymbol{Y}_{k-n}=L\left\{\boldsymbol{y}_{1}, \boldsymbol{y}_{2}, \cdots, \boldsymbol{y}_{k-n}\right\}$ is a linear space spanned by $\boldsymbol{y}_{1}, \boldsymbol{y}_{2}, \cdots, \boldsymbol{y}_{k-n}$. For convenience, we define $\tilde{\boldsymbol{x}}_{k \mid l}=\boldsymbol{x}_{k}-\boldsymbol{x}_{k \mid l}, \quad \tilde{\boldsymbol{\omega}}_{k \mid l}=\omega_{k}-\omega_{k \mid l},\left.\quad \tilde{\boldsymbol{h}}\right|_{x_{k \mid l}}=\boldsymbol{h}\left(\boldsymbol{x}_{k}\right)-\boldsymbol{h}\left(\boldsymbol{x}_{k \mid l}\right)$, $\left.(\tilde{\boldsymbol{f}}-\boldsymbol{L} \tilde{\boldsymbol{h}})\right|_{x_{k \mid l}}=\left.\tilde{\boldsymbol{f}}\right|_{x_{k \mid l}}-\left.\boldsymbol{L} \tilde{\boldsymbol{h}}\right|_{x_{k \mid l}}$, where, $k$ and $l$ take positive integers. $\boldsymbol{a} \perp \boldsymbol{b}$ means that $\boldsymbol{a}$ is uncorrelated with $\boldsymbol{b}$.

\section{PROBLEM DESCRIPTION}

Assuming the following nonlinear dynamic system with one-step compensation mechanism is considered:

$$
\begin{gathered}
\boldsymbol{x}_{k+1}=\boldsymbol{f}\left(\boldsymbol{x}_{k}\right)+\Gamma_{k} \omega_{k} \\
\boldsymbol{z}_{k}=\boldsymbol{h}\left(\boldsymbol{x}_{k}\right)+\boldsymbol{v}_{k}
\end{gathered}
$$

$$
\boldsymbol{y}_{k}=\gamma_{k} \boldsymbol{z}_{k}+\left(1-\gamma_{k}\right) \boldsymbol{z}_{k \mid k-1}
$$

where $\boldsymbol{x}_{k} \in R^{n}$ is the state, $\boldsymbol{z}_{k} \in R^{m}$ is the measurement, $\boldsymbol{y}_{k} \in R^{m}$ is the measurement received by the data processing center. Nonlinear functions $\boldsymbol{f}$ and $\boldsymbol{h}$ have been obtained. $\Gamma_{k}$ is a known constant matrix with suitable dimension. $\gamma_{k}$ is a random variable satisfied with the Bernoulli distribution and with probability $p\left(\gamma_{k}=1\right)=E\left[\gamma_{k}\right]=\alpha_{k}$, $E\left[\gamma_{k} \gamma_{k}\right]=E\left[\gamma_{k}\right]=\alpha_{k}, 0 \leq \alpha_{k} \leq 1$.

Assumption 1: $\omega_{k} \in R^{p}$ and $v_{k} \in R^{m}$ are the process and measurement noises with zero mean and satisfied with

$$
\begin{aligned}
& E\left[\omega_{k} \omega_{k-n}^{T}\right]=Q_{n}, E\left[\boldsymbol{v}_{k} \boldsymbol{v}_{k-n}^{T}\right]=R_{n} \\
& E\left[\boldsymbol{\omega}_{k} \boldsymbol{v}_{k-n}^{T}\right]=S_{n}, k \geq|n|
\end{aligned}
$$

where $|n|=0,1, \cdots, N_{0} \cdot \boldsymbol{Q}_{-n}=\boldsymbol{Q}_{n}^{T}, \boldsymbol{R}_{-n}=\boldsymbol{R}_{n}^{T}$.

Assumption 2: $\boldsymbol{x}_{0}$ is the initial state and uncorrelated with the process and measurement noises and satisfied with $E\left[\boldsymbol{x}_{0}\right]=\overline{\boldsymbol{x}}_{0}, E\left[\left(\boldsymbol{x}_{0}-\overline{\boldsymbol{x}}_{0}\right)\left(\boldsymbol{x}_{0}-\overline{\boldsymbol{x}}_{0}\right)^{T}\right]=\boldsymbol{P}_{0}$.

In this paper, our goal is mainly to design the Gaussian filter for the state $\boldsymbol{x}$ in the presence of finite-step correlated noises and packet loss, namely

$$
\begin{aligned}
& \boldsymbol{x}_{k+1 \mid k+1}=E\left[\boldsymbol{x}_{k+1} \mid \boldsymbol{Y}_{k+1}\right] \\
& \boldsymbol{P}_{k+1 \mid k+1}=E\left[\left(\boldsymbol{x}_{k+1}-\boldsymbol{x}_{k+1 \mid k+1}\right)\left(\boldsymbol{x}_{k+1}-\boldsymbol{x}_{k+1 \mid k+1}\right)^{T} \mid \boldsymbol{Y}_{k+1}\right]
\end{aligned}
$$

\section{GAUSSIAN RECURSIVE FILTER DESIGN}

For the sake of clarity, the derivation process of the filter is divided into the following three lemmas and two theorems.

Lemma 1: For system (1)-(3) and based on the Assumption 1 , the innovation $\varepsilon_{k+1}$ can be described by

$$
\boldsymbol{\varepsilon}_{k+1}=\boldsymbol{y}_{k+1}-\int \boldsymbol{h}\left(\boldsymbol{x}_{k+1}\right) N_{k+1 \mid k}^{x} d \boldsymbol{x}_{k+1}-\boldsymbol{v}_{k+1 \mid k}
$$

The corresponding covariance is as follows:

$$
\begin{aligned}
\boldsymbol{Q}_{k+1}^{\varepsilon}= & \left.\alpha_{k+1} \int \tilde{\boldsymbol{h}}\right|_{x_{k+1 \mid k}}\left(\left.\tilde{\boldsymbol{h}}\right|_{x_{k+1 \mid k}}\right)^{T} N_{k+1 \mid k}^{x} d \boldsymbol{x}_{k+1}+\alpha_{k+1} \boldsymbol{P}_{k+1 \mid k}^{v v} \\
& +\left.\alpha_{k+1} \int \tilde{\boldsymbol{h}}\right|_{x_{k+1 \mid k}} \tilde{\boldsymbol{v}}_{k+1 \mid k}^{T} N_{k+1 \mid k}^{x v} d\left[\begin{array}{c}
\boldsymbol{x}_{k+1} \\
\boldsymbol{v}_{k+1}
\end{array}\right] \\
& +\alpha_{k+1}\left(\left.\int \tilde{\boldsymbol{h}}\right|_{x_{k+1 \mid k}} \tilde{\boldsymbol{v}}_{k+1 \mid k}^{T} N_{k+1 \mid k}^{x v} d\left[\begin{array}{c}
\boldsymbol{x}_{k+1} \\
\boldsymbol{v}_{k+1}
\end{array}\right]\right)^{T}
\end{aligned}
$$

where,

$$
\begin{aligned}
& N_{k+1 \mid k}^{x}=N\left(\boldsymbol{x}_{k+1} ; \boldsymbol{x}_{k+1 \mid k}, \boldsymbol{P}_{k+1 \mid k}^{x x}\right) \\
& N_{k+1 \mid k}^{x v}=N\left(\left[\begin{array}{c}
\boldsymbol{x}_{k+1} \\
\boldsymbol{v}_{k+1}
\end{array}\right] ;\left[\begin{array}{c}
\boldsymbol{x}_{k+1 \mid k} \\
\boldsymbol{v}_{k+1 \mid k}
\end{array}\right],\left[\begin{array}{cc}
\boldsymbol{P}_{k+1 \mid k}^{x x} & \boldsymbol{P}_{k+1 \mid k}^{x v} \\
\left(\boldsymbol{P}_{k+1 \mid k}^{x v}\right)^{T} & \boldsymbol{P}_{k+1 \mid k}^{v v}
\end{array}\right]\right)
\end{aligned}
$$


In $N_{k+1 k}^{x}, \boldsymbol{x}_{k+1 \mid k}$ and $\boldsymbol{P}_{k+1 k}^{x x}$ will be calculated in Theorem 1. In $N_{k+1 \mid k}^{x v}, \boldsymbol{v}_{k+1 \mid k}, \boldsymbol{P}_{k+1 \mid k}^{x v}$ and $\boldsymbol{P}_{k+1 k}^{v v}$ will be calculated in Lemma 3.

Proof:

$$
\begin{aligned}
\boldsymbol{y}_{k+1 \mid k} & =E\left[\boldsymbol{y}_{k+1} \mid \boldsymbol{Y}_{k}\right] \\
& =E\left[\gamma_{k+1} \boldsymbol{z}_{k+1}+\left(1-\gamma_{k+1}\right) \boldsymbol{z}_{k+1 \mid k} \mid \boldsymbol{Y}_{k}\right] \\
& =\boldsymbol{z}_{k+1 \mid k}=E\left[\boldsymbol{h}\left(\boldsymbol{x}_{k+1}\right)+\boldsymbol{v}_{k+1} \mid \boldsymbol{Y}_{k}\right] \\
& =\int \boldsymbol{h}\left(\boldsymbol{x}_{k+1}\right) N_{k+1 \mid k}^{x} d \boldsymbol{x}_{k+1}+\boldsymbol{v}_{k+1 \mid k}
\end{aligned}
$$

Substituting (7) into $\boldsymbol{\varepsilon}_{k+1}=\boldsymbol{y}_{k+1}-\boldsymbol{y}_{k+1 \mid k}$ yields (5).

Using (3) to rewrite $\varepsilon_{k+1}$, we have

$$
\begin{aligned}
\boldsymbol{\varepsilon}_{k+1} & =\boldsymbol{y}_{k+1}-\boldsymbol{y}_{k+1 \mid k} \\
& =\gamma_{k+1} \boldsymbol{z}_{k+1}+\left(1-\gamma_{k+1}\right) \boldsymbol{z}_{k+1 \mid k}-\boldsymbol{z}_{k+1 \mid k} \\
& =\gamma_{k+1}\left(\boldsymbol{z}_{k+1}-\boldsymbol{z}_{k+1 \mid k}\right)=\gamma_{k+1}\left(\left.\tilde{\boldsymbol{h}}\right|_{x_{k+1 \mid k}}+\tilde{\boldsymbol{v}}_{k+1 \mid k}\right)
\end{aligned}
$$

Then

$$
\begin{aligned}
& \boldsymbol{Q}_{k+1}^{\varepsilon}=E\left[\boldsymbol{\varepsilon}_{k+1} \boldsymbol{\varepsilon}_{k+1}^{T} \mid \boldsymbol{Y}_{k}\right] \\
& =E\left[\left(\gamma_{k+1}\left(\left.\tilde{\boldsymbol{h}}\right|_{x_{k+1 \mid k}}+\tilde{\boldsymbol{v}}_{k+1 \mid k}\right)\right)\left(\gamma_{k+1}\left(\left.\tilde{\boldsymbol{h}}\right|_{x_{k+1 \mid k}}+\tilde{\boldsymbol{v}}_{k+1 \mid k}\right)\right)^{T} \mid \boldsymbol{Y}_{k}\right] \\
& =\alpha_{k+1} E\left[\left.\tilde{\boldsymbol{h}}\right|_{x_{k+1 \mid k}}\left(\left.\tilde{\boldsymbol{h}}\right|_{x_{k+1 \mid k}}\right)^{T} \mid \boldsymbol{Y}_{k}\right]+\alpha_{k+1} E\left[\tilde{\boldsymbol{v}}_{k+1 \mid k} \tilde{\boldsymbol{v}}_{k+1 \mid k}^{T} \mid \boldsymbol{Y}_{k}\right] \\
& +\alpha_{k+1} E\left[\left.\tilde{\boldsymbol{h}}\right|_{x_{k+1 \mid k}} \tilde{\boldsymbol{v}}_{k+1 \mid k}^{T} \mid \boldsymbol{Y}_{k}\right]+\alpha_{k+1} E\left[\tilde{\boldsymbol{v}}_{k+1 \mid k}\left(\tilde{\boldsymbol{h}}_{x_{k+1 \mid k}}\right)^{T} \mid \boldsymbol{Y}_{k}\right] \\
& =\left.\alpha_{k+1} \int \tilde{\boldsymbol{h}}\right|_{x_{k+1 \mid k}}\left(\left.\tilde{\boldsymbol{h}}\right|_{x_{k+1 \mid k}}\right)^{T} N_{k+1 \mid k}^{x} d \boldsymbol{x}_{k+1}+\alpha_{k+1} \boldsymbol{P}_{k+1 \mid k}^{v v} \\
& +\left.\alpha_{k+1} \int \tilde{\boldsymbol{h}}\right|_{x_{k+1 \mid k}} \tilde{\boldsymbol{v}}_{k+1 \mid k}^{T} N_{k+1 \mid k}^{x v} d\left[\begin{array}{l}
\boldsymbol{x}_{k+1} \\
\boldsymbol{v}_{k+1}
\end{array}\right] \\
& +\alpha_{k+1}\left(\left.\int \tilde{\boldsymbol{h}}\right|_{x_{k+1 \mid k}} \tilde{\boldsymbol{v}}_{k+1 \mid k}^{T} N_{k+1 \mid k}^{x v} d\left[\begin{array}{l}
\boldsymbol{x}_{k+1} \\
\boldsymbol{v}_{k+1}
\end{array}\right]\right)^{T}
\end{aligned}
$$

Lemma 1 is proved.

Lemma 2: For system (1)-(3) and based on the Assumption 1 , the estimation of $\omega_{k}$ condition on $\boldsymbol{Y}_{k-1}$ can be computed by

$$
\begin{gathered}
\boldsymbol{\omega}_{k \mid k-1}=\sum_{n=1}^{N} \boldsymbol{K}_{k \mid k-n}^{\omega} \boldsymbol{\varepsilon}_{k-n} \\
\boldsymbol{P}_{k \mid k-1}^{\omega \omega}=\boldsymbol{Q}_{0}-\sum_{n=1}^{N} \boldsymbol{K}_{k \mid k-n}^{\omega} \boldsymbol{Q}_{k-n}^{\varepsilon}\left(\boldsymbol{K}_{k \mid k-n}^{\omega}\right)^{T} \\
\boldsymbol{K}_{k \mid k-n}^{\omega}=\boldsymbol{P}_{k, k-n \mid k-n-1}^{\omega y}\left(\boldsymbol{Q}_{k-n}^{\varepsilon}\right)^{-1}
\end{gathered}
$$

where, $N=\min \left\{k, N_{0}\right\}$.

$$
\begin{aligned}
& \boldsymbol{P}_{k, k-n \mid k-n-1}^{\omega y}=\alpha_{k-n} \int \tilde{\omega}_{k \mid k-n-1}\left(\left.\tilde{\boldsymbol{h}}\right|_{x_{k-n \mid k-n-1}}\right)^{T} N_{k, k-n \mid k-n-1}^{\omega x} d\left[\begin{array}{c}
\omega_{k} \\
\boldsymbol{x}_{k-n}
\end{array}\right] \\
&+\alpha_{k-n}\left(\boldsymbol{S}_{n}-\sum_{l=n+1}^{N} \boldsymbol{K}_{k \mid k-l}^{\omega} \boldsymbol{Q}_{k-l}^{\varepsilon}\left(\boldsymbol{K}_{k-n \mid k-l}^{v}\right)^{T}\right) \\
& N_{k, k-n \mid k-n-1}^{\omega x}= \\
& N\left(\left[\begin{array}{c}
\omega_{k} \\
\boldsymbol{x}_{k-n}
\end{array}\right] ;\left[\begin{array}{c}
\omega_{k \mid k-n-1} \\
\boldsymbol{x}_{k-n \mid k-n-1}
\end{array}\right],\left[\begin{array}{cc}
\boldsymbol{P}_{k \mid k-n-1}^{\omega \omega} & \boldsymbol{P}_{k, k-n \mid k-n-1}^{\omega x} \\
\left(\boldsymbol{P}_{k, k-n \mid k-n-1}^{\omega x}\right)^{T} & \boldsymbol{P}_{k-n \mid k-n-1}^{x x}
\end{array}\right]\right)
\end{aligned}
$$

where, $\boldsymbol{x}_{k-n \mid k-n-1}$ and $\boldsymbol{P}_{k-n \mid k-n-1}^{x x}$ have been computed at $k-n$ epoch. $\omega_{k \mid k-n-1}$ and $\boldsymbol{P}_{k \mid k-n-1}^{\omega \omega}$ have been drawn in the process of calculating $\omega_{k \mid k-1}$ and $\boldsymbol{P}_{k \mid k-1}^{\omega \omega}$.

$\boldsymbol{P}_{k, k-n \mid k-n-1}^{\omega x}=$

$\int \omega_{k}\left(\left.\left(\tilde{\boldsymbol{f}}-\boldsymbol{K}_{k-n \mid k-n-1} \xi_{k-n-1} \tilde{\boldsymbol{h}}\right)\right|_{x_{k-n-1 \mid k-n-2}}\right)^{T} N_{k, k-n-1 \mid k-n-2}^{\omega x} d\left[\begin{array}{c}\omega_{k} \\ \boldsymbol{x}_{k-n-1}\end{array}\right]$

$+\left(\boldsymbol{Q}_{n+1}-\sum_{l=n+2}^{N} \boldsymbol{K}_{k \mid k-l}^{\omega} \boldsymbol{Q}_{k-l}^{\varepsilon}\left(\boldsymbol{K}_{k-n-1 \mid k-l}^{\omega}\right)^{T}\right) \Gamma_{k-n-1}^{T}$

$-\alpha_{k-n-1}\left(\boldsymbol{S}_{n+1}-\sum_{l=n+2}^{N} \boldsymbol{K}_{k \mid k-l}^{\omega} \boldsymbol{Q}_{k-l}^{\varepsilon}\left(\boldsymbol{K}_{k-n-1 \mid k-l}^{v}\right)^{T}\right) \boldsymbol{K}_{k-n \mid k-n-1}^{T}$

$$
\begin{aligned}
& N_{k, k-n-1 \mid k-n-2}^{\omega x}= \\
& N\left(\left[\begin{array}{c}
\omega_{k} \\
\boldsymbol{x}_{k-n-1}
\end{array}\right] ;\left[\begin{array}{c}
\omega_{k \mid k-n-2} \\
\boldsymbol{x}_{k-n-1 \mid k-n-2}
\end{array}\right],\left[\begin{array}{cc}
\boldsymbol{P}_{k \mid k-n-2}^{\omega \omega} & \boldsymbol{P}_{k, k-n-1 \mid k-n-2}^{\omega x} \\
\boldsymbol{P}_{k-n-1, k \mid k-n-2}^{x \omega} & \boldsymbol{P}_{k-n-1 \mid k-n-2}^{x x}
\end{array}\right]\right)
\end{aligned}
$$

To get $\omega_{k \mid k-1}$ and $\boldsymbol{P}_{k \mid k-1}^{\omega \omega}$, because $\varepsilon_{k-n}(n=1, \cdots, N)$ and $\boldsymbol{Q}_{k-n}^{\varepsilon}(n=1, \cdots, N)$ have been known at the previous epoch, we just need to compute $\boldsymbol{K}_{k \mid k-n}^{\omega}(n=1, \cdots, N)$, which is equivalent to the computation of $\boldsymbol{P}_{k, k-n \mid k-n-1}^{\omega y}(n=1, \cdots, N)$.

From (13) and (14), we know that all values in $\boldsymbol{K}_{k \mid k-n}^{\omega}$ have been calculated in $\boldsymbol{K}_{k \mid k-n-1}^{\omega}$. To get all $\boldsymbol{K}_{k \mid k-n}^{\omega}(n=1, \cdots, N)$, we can process them in the following order, $\boldsymbol{K}_{k \mid k-N}^{\omega} \rightarrow \boldsymbol{K}_{k \mid k-N+1}^{\omega} \rightarrow \cdots \rightarrow \boldsymbol{K}_{k \mid k-1}^{\omega}$.

\section{Proof:}

Using projection theorem repeatedly [27] yields

$$
\omega_{k \mid k-1}=\omega_{k \mid k-N-1}+\sum_{n=1}^{N} \boldsymbol{K}_{k \mid k-n}^{\omega} \varepsilon_{k-n}
$$


where,

$$
\boldsymbol{K}_{k \mid k-n}^{\omega}=\boldsymbol{P}_{k, k-n \mid k-n-1}^{\omega y}\left(\boldsymbol{Q}_{k-n}^{\varepsilon}\right)^{-1}
$$

Using the definition of covariance yields

$$
\begin{aligned}
\boldsymbol{P}_{k \mid k-1}^{\omega \omega} & =E\left[\left(\omega_{k}-\omega_{k \mid k-1}\right)\left(\omega_{k}-\omega_{k \mid k-1}\right)^{T} \mid \boldsymbol{Y}_{k-1}\right] \\
& =E\left[\omega_{k}\left(\omega_{k}-\omega_{k \mid k-N-1}-\sum_{n=1}^{N} \boldsymbol{K}_{k \mid k-n}^{\omega} \varepsilon_{k-n}\right)^{T}\right] \\
& =\boldsymbol{P}_{k \mid k-N-1}^{\omega \omega}-\sum_{n=1}^{N} E\left[\omega_{k} \varepsilon_{k-n}^{T}\right]\left(\boldsymbol{K}_{k \mid k-n}^{\omega}\right)^{T}
\end{aligned}
$$

From (16), we have

$$
\boldsymbol{P}_{k, k-n \mid k-n-1}^{\omega y}=E\left[\omega_{k} \boldsymbol{\varepsilon}_{k-n}^{T}\right]=\boldsymbol{K}_{k \mid k-n}^{\omega} \boldsymbol{Q}_{k-n}^{\varepsilon}
$$

Because of $\omega_{k} \perp \boldsymbol{Y}_{k-N-1}$, we have

$$
\begin{gathered}
\boldsymbol{\omega}_{k \mid k-N-1}=E\left[\boldsymbol{\omega}_{k} \mid \boldsymbol{Y}_{k-N-1}\right]=\mathbf{0} \\
\boldsymbol{P}_{k \mid k-N-1}^{\omega \omega}=E\left[\omega_{k}\left(\boldsymbol{\omega}_{k}-\boldsymbol{\omega}_{k \mid k-N-1}\right)^{T} \mid \boldsymbol{Y}_{k-N-1}\right] \\
=E\left[\omega_{k} \boldsymbol{\omega}_{k}^{T} \mid \boldsymbol{Y}_{k-N-1}\right]=\boldsymbol{Q}_{0}
\end{gathered}
$$

Substituting (18), (19), and (20) into (15) and (17) gets (10) and (11).

In (16),

$$
\begin{aligned}
\boldsymbol{P}_{k, k-n \mid k-n-1}^{\omega y} & =E\left[\tilde{\boldsymbol{\omega}}_{k \mid k-n-1} \tilde{\boldsymbol{y}}_{k-n \mid k-n-1}^{T} \mid \boldsymbol{Y}_{k-n-1}\right] \\
= & \alpha_{k-n} E\left[\tilde{\boldsymbol{\omega}}_{k \mid k-n-1}\left(\left.\tilde{\boldsymbol{h}}\right|_{x_{k-n \mid k-n-1}}+\tilde{\boldsymbol{v}}_{k-n \mid k-n-1}\right)^{T} \mid \boldsymbol{Y}_{k-n-1}\right] \\
= & \alpha_{k-n} E\left[\tilde{\boldsymbol{\omega}}_{k \mid k-n-1}\left(\left.\tilde{\boldsymbol{h}}\right|_{x_{k-n \mid k-n-1}}\right)^{T} \mid \boldsymbol{Y}_{k-n-1}\right] \\
& +\alpha_{k-n} E\left[\tilde{\boldsymbol{\omega}}_{k \mid k-n-1} \tilde{\boldsymbol{v}}_{k-n \mid k-n-1}^{T} \mid \boldsymbol{Y}_{k-n-1}\right] \\
= & \alpha_{k-n} \int \tilde{\boldsymbol{\omega}}_{k \mid k-n-1}\left(\left.\tilde{\boldsymbol{h}}\right|_{x_{k-n \mid k-n-1}}\right)^{T} N_{k, k-n \mid k-n-1}^{\omega x} d\left[\begin{array}{c}
\boldsymbol{\omega}_{k} \\
\boldsymbol{x}_{k-n}
\end{array}\right] \\
& +\alpha_{k-n} \boldsymbol{P}_{k, k-n \mid k-n-1}^{\omega D}
\end{aligned}
$$

In the following, we calculate $\boldsymbol{P}_{k, k-n \mid k-n-1}^{\omega v}$ and $N_{k, k-n \mid k-n-1}^{\omega x}$. From Lemma 3, we have

$$
\begin{aligned}
\tilde{\boldsymbol{v}}_{k-n \mid k-n-1} & =\boldsymbol{v}_{k-n}-\boldsymbol{v}_{k-n \mid k-n-1} \\
& =\boldsymbol{v}_{k-n}-\sum_{l=n+1}^{n+N} \boldsymbol{K}_{k-n \mid k-l}^{\nu} \boldsymbol{\varepsilon}_{k-l}
\end{aligned}
$$

Because of $\omega_{k} \perp v_{k-l}(l>N)$ and from (18), we have

$$
\begin{aligned}
\boldsymbol{P}_{k, k-n \mid k-n-1}^{\omega \nu} & =E\left[\tilde{\boldsymbol{\omega}}_{k \mid k-n-1} \tilde{\boldsymbol{v}}_{k-n \mid k-n-1}^{T} \mid \boldsymbol{Y}_{k-n-1}\right] \\
& =E\left[\omega_{k} \tilde{\boldsymbol{V}}_{k-n \mid k-n-1}^{T} \mid \boldsymbol{Y}_{k-n-1}\right] \\
& =\boldsymbol{S}_{n}-\sum_{l=n+1}^{N} \boldsymbol{K}_{k \mid k-l}^{\omega} \boldsymbol{Q}_{k-l}^{\varepsilon}\left(\boldsymbol{K}_{k-n \mid k-l}^{v}\right)^{T}
\end{aligned}
$$

In $N_{k, k-n \mid k-n-1}^{\omega x}$, based on Theorem 1, $\boldsymbol{P}_{k, k-n \mid k-n-1}^{\omega x}$ can be computed as follows.

$$
\begin{aligned}
\tilde{\boldsymbol{x}}_{k-n \mid k-n-1}=\boldsymbol{x}_{k-n}-\boldsymbol{x}_{k-n \mid k-n-1} \\
=\boldsymbol{f}\left(\boldsymbol{x}_{k-n-1}\right)+\omega_{k-n-1}-\left(\boldsymbol{x}_{k-n \mid k-n-2}-\boldsymbol{K}_{k-n \mid k-n-1} \varepsilon_{k-n-1}\right) \\
=\left.\left(\tilde{\boldsymbol{f}}-\boldsymbol{K}_{k-n \mid k-n-1} \gamma_{k-n-1} \tilde{\boldsymbol{h}}\right)\right|_{x_{k-n-1 \mid k-n-2}}+\tilde{\boldsymbol{\omega}}_{k-n-1 \mid k-n-2} \\
\quad-\boldsymbol{K}_{k-n \mid k-n-1} \gamma_{k-n-1} \tilde{\boldsymbol{\omega}}_{k-n-1 \mid k-n-2}
\end{aligned}
$$

$$
\begin{aligned}
& \boldsymbol{P}_{k, k-n \mid k-n-1}^{\omega x}=E\left[\tilde{\omega}_{k \mid k-n-1} \tilde{\boldsymbol{x}}_{k-n \mid k-n-1}^{T} \mid \boldsymbol{Y}_{k-n-1}\right] \\
& =E\left[\omega_{k} \tilde{\boldsymbol{x}}_{k-n \mid k-n-1}^{T} \mid \boldsymbol{Y}_{k-n-1}\right] \\
& \left.=E\left[\begin{array}{l}
\left.\left(\tilde{\boldsymbol{f}}-\boldsymbol{K}_{k-n \mid k-n-1} \gamma_{k-n-1} \tilde{\boldsymbol{h}}\right)\right|_{x_{k-n \mid k-n-1}} \\
+\Gamma_{k-n-1} \tilde{\boldsymbol{\omega}}_{k-n-1 \mid k-n-2} \\
-\boldsymbol{K}_{k-n \mid k-n-1} \gamma_{k-n-1} \tilde{\boldsymbol{v}}_{k-n-1 \mid k-n-2}
\end{array}\right)^{T} \mid \boldsymbol{Y}_{k-n-2}\right] \\
& =E\left[\boldsymbol{\omega}_{k}\left(\left.\left(\tilde{\boldsymbol{f}}-\boldsymbol{K}_{k-n \mid k-n-1} \gamma_{k-n-1} \tilde{\boldsymbol{h}}\right)\right|_{x_{k-n-1 \mid k-n-2}}\right)^{T} \mid \boldsymbol{Y}_{k-n-2}\right] \\
& +E\left[\omega_{k} \tilde{\omega}_{k-n-1 \mid k-n-2}^{T} \mid \boldsymbol{Y}_{k-n-2}\right] \Gamma_{k-n-1}^{T} \\
& -\alpha_{k-n-1} E\left[\omega_{k} \tilde{\boldsymbol{v}}_{k-n-1 \mid k-n-2}^{T} \mid \boldsymbol{Y}_{k-n-2}\right] \boldsymbol{K}_{k-n \mid k-n-1}^{T} \\
& =\int \omega_{k}\left(\left.\left(\tilde{\boldsymbol{f}}-\boldsymbol{K}_{k-n \mid k-n-1} \alpha_{k-n-1} \tilde{\boldsymbol{n}}\right)\right|_{x_{k-n-n-1 \mid k-n-2}}\right)^{T} N_{k, k-n-1 \mid k-n-2}^{\omega x} d\left[\begin{array}{c}
\omega_{k} \\
\boldsymbol{x}_{k-n-1}
\end{array}\right] \\
& +\boldsymbol{P}_{k, k-n-1 \mid k-n-2}^{\omega \omega} \Gamma_{k-n-1}^{T}-\alpha_{k-n-1} \boldsymbol{P}_{k, k-n-1 \mid k-n-2}^{\omega \nu} \boldsymbol{K}_{k-n \mid k-n-1}^{T}
\end{aligned}
$$

From (10), we have

$$
\begin{aligned}
\tilde{\omega}_{k-n-1 \mid k-n-2} & =\omega_{k-n-1}-\omega_{k-n-1 \mid k-n-2} \\
& =\omega_{k-n-1}-\sum_{l=n+2}^{n+N} \boldsymbol{K}_{k-n-1 \mid k-l}^{\omega} \varepsilon_{k-l}
\end{aligned}
$$

In (25), because of $\omega_{k} \perp \varepsilon_{k-l}(l>N)$, we have

$$
\begin{aligned}
\boldsymbol{P}_{k, k-n-1 \mid k-n-2}^{\omega \omega} & =E\left[\tilde{\omega}_{k \mid k-n-2} \tilde{\omega}_{k-n-1 \mid k-n-2}^{T} \mid \boldsymbol{Y}_{k-n-2}\right] \\
& =E\left[\omega_{k} \tilde{\omega}_{k-n-1 \mid k-n-2}^{T} \mid \boldsymbol{Y}_{k-n-2}\right] \\
& =\boldsymbol{Q}_{n+1}-\sum_{l=n+2}^{N} \boldsymbol{K}_{k \mid k-l}^{\omega} \boldsymbol{Q}_{k-l}^{\varepsilon}\left(\boldsymbol{K}_{k-n-1 \mid k-l}^{\omega}\right)^{T}
\end{aligned}
$$

$\boldsymbol{P}_{k, k-n-1 \mid k-n-2}^{\omega v}$ in (25) can be calculated like $\boldsymbol{P}_{k, k-n \mid k-n-1}^{\omega v}$ in (23), where $n$ in $\boldsymbol{P}_{k, k-n \mid k-n-1}^{\omega v}$ need to be substituted by $n+1$. 
Lemma 3: For system (1)-(3) and based on the Assumption 1 , the mean and covariance of $\boldsymbol{v}_{k+1}$ in condition $\boldsymbol{Y}_{k}$ can be computed by

$$
\begin{gathered}
\boldsymbol{v}_{k+1 \mid k}=\sum_{n=1}^{N} \boldsymbol{K}_{k+1 \mid k+1-n}^{v} \boldsymbol{\varepsilon}_{k+1-n} \\
\boldsymbol{P}_{k+1 \mid k}^{v v}=\boldsymbol{R}_{0}-\sum_{n=1}^{N} \boldsymbol{K}_{k+1 \mid k+1-n}^{v} \boldsymbol{Q}_{k+1-n}^{\varepsilon}\left(\boldsymbol{K}_{k+1 \mid k+1-n}^{v}\right)^{T} \\
\boldsymbol{K}_{k+1 \mid k+1-n}^{v}=\boldsymbol{P}_{k+1, k+1-n \mid k-n}^{v y}\left(\boldsymbol{Q}_{k+1-n}^{\varepsilon}\right)^{-1}
\end{gathered}
$$

where,

$$
\begin{aligned}
& \quad \boldsymbol{P}_{k+1, k+1-n \mid k-n}^{v y} \\
& =\alpha_{k+1-n} \int \tilde{\boldsymbol{v}}_{k+1 \mid k-n}\left(\tilde{\boldsymbol{h}}_{x_{k+1-n \mid k-n}}\right)^{T} N_{k+1, k+1-n \mid k-n}^{v x} d\left[\begin{array}{c}
\boldsymbol{v}_{k+1} \\
\boldsymbol{x}_{k+1-n}
\end{array}\right] \\
& \quad+\alpha_{k+1-n}\left(\boldsymbol{R}_{n}-\sum_{l=n}^{N-1} \boldsymbol{K}_{k+1 \mid k-l}^{v} \boldsymbol{Q}_{k-l}^{\varepsilon}\left(\boldsymbol{K}_{k+1-n \mid k-l}^{v}\right)^{T}\right) \\
& N_{k+1, k+1-n \mid k-n}^{v x} \\
& =N\left(\left[\begin{array}{c}
\boldsymbol{v}_{k+1} \\
\boldsymbol{x}_{k+1-n}
\end{array}\right] ;\left[\begin{array}{c}
\boldsymbol{v}_{k+1 \mid k-n} \\
\boldsymbol{x}_{k+1-n \mid k-n}
\end{array}\right],\left[\begin{array}{cc}
\boldsymbol{P}_{k+1 \mid k-n}^{v v} & \boldsymbol{P}_{k+1, k+1-n \mid k-n}^{v x} \\
\left(\boldsymbol{P}_{k+1, k+1-n \mid k-n}^{v x}\right)^{T} & \boldsymbol{P}_{k+1-n \mid k-n}^{x x}
\end{array}\right]\right)
\end{aligned}
$$

where, $\quad \boldsymbol{x}_{k+1-n \mid k-n}$ and $\boldsymbol{P}_{k+1-n \mid k-n}^{x x}$ have been computed at $k+1-n$ epoch. $v_{k+1 \mid k-n}$ and $\boldsymbol{P}_{k+1 \mid k-n}^{v v}$ have been drawn in the process of calculating $\boldsymbol{v}_{k+1 \mid k}$ and $\boldsymbol{P}_{k+1 \mid k}^{v v}$.

$$
\begin{aligned}
& \boldsymbol{P}_{k+1, k+1-n \mid k-n}^{v x}= \\
& \int \boldsymbol{v}_{k+1}\left(\left.\left(\tilde{\boldsymbol{f}}-\boldsymbol{K}_{k+1-n \mid k-n} \gamma_{k-n} \tilde{\boldsymbol{h}}\right)\right|_{k_{k-n \mid k-n-1}}\right)^{T} N_{k+1, k-n \mid k-n-1}^{v x} d\left[\begin{array}{c}
\boldsymbol{v}_{k+1} \\
\boldsymbol{x}_{k-n}
\end{array}\right] \\
& +\left(\boldsymbol{S}_{-(n+1)}^{T}-\sum_{l=n+1}^{N-1} \boldsymbol{K}_{k+1 \mid k-l}^{v} \boldsymbol{Q}_{k-l}^{\varepsilon}\left(\boldsymbol{K}_{k-n \mid k-l}^{\omega}\right)^{T}\right) \Gamma_{k-n}^{T} \\
& -\alpha_{k-n}\left(\boldsymbol{R}_{n+1}-\sum_{l=n+1}^{N-1} \boldsymbol{K}_{k+1 \mid k-l}^{v} \boldsymbol{Q}_{k-l}^{\varepsilon}\left(\boldsymbol{K}_{k-n \mid k-l}^{v}\right)^{T}\right) \boldsymbol{K}_{k+1-n \mid k-n}^{T}
\end{aligned}
$$

$$
\begin{aligned}
& N_{k+1, k-n \mid k-n-1}^{v x} \\
& =N\left(\left[\begin{array}{l}
\boldsymbol{v}_{k+1} \\
\boldsymbol{x}_{k-n}
\end{array}\right] ;\left[\begin{array}{l}
\boldsymbol{v}_{k+1 \mid k-n-1} \\
\boldsymbol{x}_{k-n \mid k-n-1}
\end{array}\right],\left[\begin{array}{cc}
\boldsymbol{P}_{k+1 \mid k-n-1}^{v v} & \boldsymbol{P}_{k+1, k-n \mid k-n-1}^{v x} \\
\left(\boldsymbol{P}_{k+1, k-n \mid k-n-1}^{v x}\right)^{T} & \boldsymbol{P}_{k-n \mid k-n-1}^{x x}
\end{array}\right]\right)
\end{aligned}
$$

To get $v_{k+1 \mid k}$ and $\boldsymbol{P}_{k+1 \mid k}^{v v}$, based on (31) and (32) and the analysis process in Lemma 2, we can calculate the gain matrix in the following order,

$\boldsymbol{K}_{k+1 \mid k-N+1}^{v} \rightarrow \boldsymbol{K}_{k+1 \mid k-N+2}^{v} \rightarrow \cdots \rightarrow \boldsymbol{K}_{k+1 \mid k}^{v}$.
Proof:

Using the projection theorem repeatedly yields

$$
\boldsymbol{v}_{k+1 \mid k}=\boldsymbol{v}_{k+1 \mid k-N}+\sum_{n=1}^{N} \boldsymbol{K}_{k+1 \mid k+1-n}^{v} \boldsymbol{\varepsilon}_{k+1-n}
$$

and

$$
\boldsymbol{K}_{k+1 \mid k+1-n}^{v}=\boldsymbol{P}_{k+1, k+1-n \mid k-n}^{v y}\left(\boldsymbol{Q}_{k+1-n}^{\varepsilon}\right)^{-1}
$$

Using the definition of covariance yields

$$
\begin{aligned}
& \boldsymbol{P}_{k+1 \mid k}^{v v}=E\left[\left(\boldsymbol{v}_{k+1}-\boldsymbol{v}_{k+1 \mid k}\right)\left(\boldsymbol{v}_{k+1}-\boldsymbol{v}_{k+1 \mid k}\right)^{T} \mid \boldsymbol{Y}_{k}\right] \\
& =E\left[\boldsymbol{v}_{k+1}\left(\boldsymbol{v}_{k+1}-\boldsymbol{v}_{k+1 \mid k}\right)^{T} \mid \boldsymbol{Y}_{k}\right] \\
& =E\left[\boldsymbol{v}_{k+1}\left(\boldsymbol{v}_{k+1}-\boldsymbol{v}_{k+1 \mid k-N}-\sum_{n=1}^{N} \boldsymbol{K}_{k+1 \mid k+1-n}^{v} \boldsymbol{\varepsilon}_{k+1-n}\right)^{T}\right] \\
& =\boldsymbol{P}_{k+1 \mid k-N}^{v v}-\sum_{n=1}^{N} E\left[\boldsymbol{v}_{k+1} \boldsymbol{\varepsilon}_{k+1-n}^{T}\right]\left(\boldsymbol{K}_{k+1 \mid k+1-n}^{v}\right)^{T}
\end{aligned}
$$

From (34), we have

$$
\boldsymbol{P}_{k+1, k+1-n \mid k-n}^{v y}=E\left[\boldsymbol{v}_{k+1} \boldsymbol{\varepsilon}_{k+1-n}^{T}\right]=\boldsymbol{K}_{k+1 \mid k+1-n}^{v} \boldsymbol{Q}_{k+1-n}^{\varepsilon}
$$

Because of $v_{k+1} \perp \boldsymbol{Y}_{k-N}$, we have

$$
\begin{array}{r}
\boldsymbol{v}_{k+1 \mid k-N}=E\left[\boldsymbol{v}_{k+1} \mid \boldsymbol{Y}_{k-N}\right]=\mathbf{0} \\
\boldsymbol{P}_{k+1 \mid k-N}^{v v}=E\left[\boldsymbol{v}_{k+1} \tilde{\boldsymbol{v}}_{k+1 \mid k-N}^{T} \mid \boldsymbol{Y}_{k-N}\right] \\
=E\left[\boldsymbol{v}_{k+1} \boldsymbol{v}_{k+1}^{T} \mid \boldsymbol{Y}_{k-N}\right]=\boldsymbol{R}_{0}
\end{array}
$$

Substituting (36), (37), and (38) into (33) and (35), we get (28) and (29).

In (34),

$$
\begin{aligned}
& \boldsymbol{P}_{k+1, k+1-n \mid k-n}^{v y} \\
= & E\left[\tilde{\boldsymbol{v}}_{k+1 \mid k-n} \tilde{\boldsymbol{y}}_{k+1-n \mid k-n}^{T} \mid Y_{k-n}\right] \\
= & \alpha_{k+1-n} E\left[\tilde{\boldsymbol{v}}_{k+1 \mid k-n}\left(\left.\tilde{\boldsymbol{h}}\right|_{x_{k+1-n \mid k-n}}+\tilde{\boldsymbol{v}}_{k+1-n \mid k-n}\right)^{T} \mid \boldsymbol{Y}_{k-n}\right] \\
= & \alpha_{k+1-n} E\left[\tilde{\boldsymbol{v}}_{k+1 \mid k-n}\left(\left.\tilde{\boldsymbol{h}}\right|_{x_{k+1-n \mid k-n}}\right)^{T} \mid \boldsymbol{Y}_{k-n}\right] \\
& +\alpha_{k+1-n} E\left[\tilde{\boldsymbol{v}}_{k+1 \mid k-n} \tilde{\boldsymbol{v}}_{k+1-n \mid k-n}^{T} \mid \boldsymbol{Y}_{k-n}\right] \\
= & \alpha_{k+1-n} \int \tilde{\boldsymbol{v}}_{k+1 \mid k-n}\left(\left.\tilde{\boldsymbol{h}}\right|_{x_{k+1-n \mid k-n}}\right)^{T} N_{k+1, k+1-n \mid k-n}^{v x} d\left[\begin{array}{c}
\boldsymbol{v}_{k+1} \\
\boldsymbol{x}_{k+1-n}
\end{array}\right] \\
& +\alpha_{k+1-n} \boldsymbol{P}_{k+1, k+1-n \mid k-n}^{v v}
\end{aligned}
$$

Next, we calculate $\boldsymbol{P}_{k+1, k+1-n \mid k-n}^{v v}$ and $N_{k+1, k+1-n \mid k-n}^{v x}$. 
From (28), we have

$$
\begin{aligned}
\tilde{\boldsymbol{v}}_{k+1-n \mid k-n} & =\boldsymbol{v}_{k+1-n}-\boldsymbol{v}_{k+1-n \mid k-n} \\
& =\boldsymbol{v}_{k+1-n}-\sum_{l=n}^{n+N-1} \boldsymbol{K}_{k+1-n \mid k-l}^{v} \boldsymbol{\varepsilon}_{k-l}
\end{aligned}
$$

Because of $v_{k+1} \perp \varepsilon_{k-l}(l>N-1)$ and from (36), we have

$$
\begin{aligned}
\boldsymbol{P}_{k+1, k+1-n \mid k-n}^{v v} & =E\left[\tilde{\boldsymbol{v}}_{k+1 \mid k-n} \tilde{\boldsymbol{v}}_{k+1-n \mid k-n}^{T} \mid \boldsymbol{Y}_{k-n}\right] \\
& =E\left[\boldsymbol{v}_{k+1} \tilde{\boldsymbol{v}}_{k+1-n \mid k-n}^{T} \mid \boldsymbol{Y}_{k-n}\right] \\
& =\boldsymbol{R}_{n}-\sum_{l=n}^{N-1} \boldsymbol{K}_{k+1 \mid k-l}^{v} \boldsymbol{Q}_{k-l}^{\varepsilon}\left(\boldsymbol{K}_{k+1-n \mid k-l}^{v}\right)^{T}
\end{aligned}
$$

In $N_{k+1, k+1-n \mid k-n}^{v x}$, based on Theorem $1, \boldsymbol{P}_{k+1, k+1-n \mid k-n}^{v x}$ can be calculated as follows.

$$
\begin{aligned}
& \boldsymbol{P}_{k+1, k+1-n \mid k-n}^{v x}=E\left[\tilde{\boldsymbol{v}}_{k+1 \mid k-n} \tilde{\boldsymbol{x}}_{k+1-n \mid k-n}^{T} \mid \boldsymbol{Y}_{k-n}\right] \\
& =E\left[\boldsymbol{v}_{k+1} \tilde{\boldsymbol{x}}_{k+1-n \mid k-n}^{T} \mid \boldsymbol{Y}_{k-n}\right] \\
& =E\left[\boldsymbol{v}_{k+1}\left(\begin{array}{l}
\left.\left(\tilde{\boldsymbol{f}}-\boldsymbol{K}_{k+1-n \mid k-n} \gamma_{k-n} \tilde{\boldsymbol{h}}\right)\right|_{x_{k-n \mid k-n-1}} \\
+\boldsymbol{\Gamma}_{k-n} \tilde{\boldsymbol{\omega}}_{k-n \mid k-n-1} \\
-\boldsymbol{K}_{k+1-n \mid k-n} \gamma_{k-n} \tilde{\boldsymbol{v}}_{k-n \mid k-n-1}
\end{array}\right)^{T} \mid \boldsymbol{Y}_{k-n-1}\right] \\
& =E\left[\boldsymbol{v}_{k+1}\left(\left.\left(\tilde{\boldsymbol{f}}-\boldsymbol{K}_{k+1-n \mid k-n} \gamma_{k-n} \tilde{\boldsymbol{h}}\right)\right|_{x_{k-n \mid k-n-1}}\right)^{T} \mid \boldsymbol{Y}_{k-n-1}\right] \\
& +E\left[v_{k+1} \tilde{\omega}_{k-n \mid k-n-1}^{T} \mid \boldsymbol{Y}_{k-n-1}\right] \Gamma_{k-n}^{T} \\
& -\alpha_{k-n} E\left[\boldsymbol{v}_{k+1} \tilde{\boldsymbol{v}}_{k-n \mid k-n-1}^{T} \mid \boldsymbol{Y}_{k-n-1}\right] \boldsymbol{K}_{k+1-n \mid k-n}^{T} \\
& =\int \boldsymbol{v}_{k+1}\left(\left.\left(\tilde{\boldsymbol{f}}-\boldsymbol{K}_{k+1-n \mid k-n} \alpha_{k-n} \tilde{\boldsymbol{h}}\right)\right|_{x_{k-n \mid k-n-1}}\right)^{T} N_{k+1, k-n \mid k-n-1}^{v x} d\left[\begin{array}{l}
\boldsymbol{v}_{k+1} \\
\boldsymbol{x}_{k-n}
\end{array}\right] \\
& +\boldsymbol{P}_{k+1, k-n \mid k-n-1}^{v \omega} \Gamma_{k-n}^{T}-\alpha_{k-n} \boldsymbol{P}_{k+1, k-n \mid k-n-1}^{v v} \boldsymbol{K}_{k+1-n \mid k-n}^{T}
\end{aligned}
$$

From (10), we have

$$
\begin{aligned}
\tilde{\omega}_{k-n \mid k-n-1} & =\omega_{k-n}-\omega_{k-n \mid k-n-1} \\
& =\omega_{k-n}-\sum_{l=n+1}^{n+N} \boldsymbol{K}_{k-n \mid k-l}^{\omega} \boldsymbol{\varepsilon}_{k-l}
\end{aligned}
$$

In (42), because of $v_{k+1} \perp \omega_{k-l}(l>N-1)$, we have

$$
\begin{aligned}
\boldsymbol{P}_{k+1, k-n \mid k-n-1}^{v \omega} & =E\left[\tilde{\boldsymbol{v}}_{k+1 \mid k-n-1} \tilde{\boldsymbol{\omega}}_{k-n \mid k-n-1}^{T} \mid \boldsymbol{Y}_{k-n-1}\right] \\
& =E\left[\boldsymbol{v}_{k+1} \tilde{\boldsymbol{\omega}}_{k-n \mid k-n-1}^{T} \mid \boldsymbol{Y}_{k-n-1}\right] \\
& =\boldsymbol{S}_{-(n+1)}^{T}-\sum_{l=n+1}^{N-1} \boldsymbol{K}_{k+1 \mid k-l}^{v} \boldsymbol{Q}_{k-l}^{\varepsilon}\left(\boldsymbol{K}_{k-n \mid k-l}^{\omega}\right)^{T}
\end{aligned}
$$

$\boldsymbol{P}_{k+1, k-n \mid k-n-1}^{v v}$ in (42) can be calculated like $\boldsymbol{P}_{k+1, k+1-n \mid k-n}^{v v}$ in (41), where $n$ in $\boldsymbol{P}_{k+1, k+1-n \mid k-n}^{v v}$ need to be substituted by $n+1$. Theorem 1: For system (1)-(3) and Assumption 1, the Gaussian recursive one-step predictor is given by

$$
\boldsymbol{x}_{k+1 \mid k}=\boldsymbol{x}_{k+1 \mid k-1}+\boldsymbol{K}_{k+1 \mid k} \boldsymbol{\varepsilon}_{k}
$$

$$
\begin{gathered}
\boldsymbol{P}_{k+1 \mid k}^{x x}=\boldsymbol{P}_{k+1 \mid k-1}^{x x}-\boldsymbol{K}_{k+1 \mid k} \boldsymbol{Q}_{k}^{\varepsilon} \boldsymbol{K}_{k+1 \mid k}^{T} \\
\boldsymbol{K}_{k+1 \mid k}=\boldsymbol{P}_{k+1, k \mid k-1}^{x y}\left(\boldsymbol{Q}_{k}^{\varepsilon}\right)^{-1}
\end{gathered}
$$

where

$$
\boldsymbol{x}_{k+1 \mid k-1}=\int \boldsymbol{f}\left(\boldsymbol{x}_{k}\right) N_{k \mid k-1}^{x} d \boldsymbol{x}_{k}+\Gamma_{k} \omega_{k \mid k-1}
$$

$$
\begin{aligned}
\boldsymbol{P}_{k+1 \mid k-1}^{x x} & =\left.\int \tilde{\boldsymbol{f}}\right|_{x_{k \mid k-1}}\left(\left.\tilde{\boldsymbol{f}}\right|_{x_{k \mid k-1}}\right)^{T} N_{k \mid k-1}^{x} d \boldsymbol{x}_{k} \\
& +\left.\int \tilde{\boldsymbol{f}}\right|_{x_{k \mid k-1}} \tilde{\boldsymbol{\omega}}_{k \mid k-1}^{T} \Gamma_{k}^{T} N_{k \mid k-1}^{x \omega} d\left[\begin{array}{c}
\boldsymbol{x}_{k} \\
\omega_{k}
\end{array}\right] \\
& +\left(\left.\int \tilde{\boldsymbol{f}}\right|_{x_{k \mid k-1}} \tilde{\boldsymbol{\omega}}_{k \mid k-1}^{T} \Gamma_{k}^{T} N_{k \mid k-1}^{x \omega} d\left[\begin{array}{c}
\boldsymbol{x}_{k} \\
\boldsymbol{\omega}_{k}
\end{array}\right]\right)^{T}+\Gamma_{k} \boldsymbol{P}_{k \mid k-1}^{\omega \omega} \Gamma_{k}^{T} \\
\boldsymbol{K}_{k+1 \mid k}= & \left(\begin{array}{l}
\left.\alpha_{k} \int \tilde{\boldsymbol{f}}\right|_{x_{k \mid k-1}}\left(\left.\tilde{\boldsymbol{h}}\right|_{x_{k \mid k-1}}\right)^{T} N_{k \mid k-1}^{x} d \boldsymbol{x}_{k} \\
+\left.\alpha_{k} \int \tilde{\boldsymbol{f}}\right|_{x_{k \mid k-1}} \tilde{\boldsymbol{v}}_{k \mid k-1}^{T} N_{k \mid k-1}^{x v} d\left[\begin{array}{l}
\boldsymbol{x}_{k} \\
\boldsymbol{v}_{k}
\end{array}\right]+\Gamma_{k} \boldsymbol{P}_{k \mid k-1}^{\omega y}
\end{array}\right)\left(\boldsymbol{Q}_{k}^{\varepsilon}\right)^{-1}
\end{aligned}
$$

In (45)-(49), $\boldsymbol{\varepsilon}_{k}, \boldsymbol{Q}_{k}^{\varepsilon}, \quad N_{k \mid k-1}^{x}$, and $N_{k \mid k-1}^{x v}$ have been computed at the last epoch. In $N_{k \mid k-1}^{x \omega}, \omega_{k \mid k-1}, \boldsymbol{P}_{k \mid k-1}^{\omega \omega}$, and $\boldsymbol{P}_{k \mid k-1}^{x \omega}$ are calculated in Lemma 2. In $\boldsymbol{K}_{k+1 \mid k}, \boldsymbol{P}_{k \mid k-1}^{\omega y}$ can be obtained from (13).

Proof:

From GASF in [11], we have (45)-(47), and

$$
\begin{gathered}
\boldsymbol{x}_{k+1 \mid k-1}=E\left[\boldsymbol{f}\left(\boldsymbol{x}_{k}\right)+\Gamma_{k} \omega_{k} \mid \boldsymbol{Y}_{k-1}\right] \\
=\int \boldsymbol{f}\left(\boldsymbol{x}_{k}\right) N_{k \mid k-1}^{x} d \boldsymbol{x}_{k}+\Gamma_{k} \boldsymbol{\omega}_{k \mid k-1} \\
\boldsymbol{P}_{k+1 \mid k-1}^{x x}=E\left[\left(\boldsymbol{x}_{k+1}-\boldsymbol{x}_{k+1 \mid k-1}\right)\left(\boldsymbol{x}_{k+1}-\boldsymbol{x}_{k+1 \mid k-1}\right)^{T} \mid \boldsymbol{Y}_{k-1}\right] \\
=E\left[\left(\left.\tilde{\boldsymbol{f}}\right|_{x_{k \mid k-1}}+\Gamma_{k} \tilde{\boldsymbol{\omega}}_{k \mid k-1}\right)\left(\left.\tilde{\boldsymbol{f}}\right|_{x_{k \mid k-1}}+\Gamma_{k} \tilde{\boldsymbol{\omega}}_{k \mid k-1}\right)^{T} \mid \boldsymbol{Y}_{k-1}\right] \\
=\left.\int \tilde{\boldsymbol{f}}\right|_{x_{k \mid k-1}}\left(\left.\tilde{\boldsymbol{f}}\right|_{x_{k \mid k-1}}\right)^{T} N_{k \mid k-1}^{x} d \boldsymbol{x}_{k}+\left.\int \tilde{\boldsymbol{f}}\right|_{x_{k \mid k-1}} \tilde{\boldsymbol{\omega}}_{k \mid k-1}^{T} \Gamma_{k}^{T} N_{k \mid k-1}^{x \omega} d\left[\begin{array}{c}
\boldsymbol{x}_{k} \\
\omega_{k}
\end{array}\right] \\
+\left(\left.\int \tilde{\boldsymbol{f}}\right|_{x_{k \mid k-1}} \tilde{\boldsymbol{\omega}}_{k \mid k-1}^{T} \Gamma_{k}^{T} N_{k \mid k-1}^{x \omega} d\left[\begin{array}{c}
\boldsymbol{x}_{k} \\
\omega_{k}
\end{array}\right]\right)^{T}+\Gamma_{k} \boldsymbol{P}_{k \mid k-1}^{\omega \omega} \Gamma_{k}^{T}
\end{gathered}
$$


In (47),

$$
\begin{aligned}
& \boldsymbol{P}_{k+1, k \mid k-1}^{x y}=E\left[\tilde{\boldsymbol{x}}_{k+1 \mid k-1} \boldsymbol{\varepsilon}_{k}^{T} \mid Y_{k-1}\right] \\
& =E\left[\left(\left.\tilde{\boldsymbol{f}}\right|_{x_{k \mid k-1}}+\Gamma_{k} \tilde{\boldsymbol{\omega}}_{k \mid k-1}\right) \boldsymbol{\varepsilon}_{k}^{T} \mid \boldsymbol{Y}_{k-1}\right] \\
& =\alpha_{k} E\left[\left.\tilde{\boldsymbol{f}}\right|_{x_{k \mid k-1}}\left(\left.\tilde{\boldsymbol{h}}\right|_{x_{k \mid k-1}}+\tilde{\boldsymbol{v}}_{k \mid k-1}\right)^{T} \mid \boldsymbol{Y}_{k-1}\right]+\Gamma_{k} \boldsymbol{P}_{k \mid k-1}^{\omega y} \\
& =\left.\alpha_{k} \int \tilde{\boldsymbol{f}}\right|_{x_{k \mid k-1}}\left(\left.\tilde{\boldsymbol{h}}\right|_{x_{k \mid k-1}}\right)^{T} N_{k \mid k-1}^{x} d \boldsymbol{x}_{k} \\
& \quad+\left.\alpha_{k} \int \tilde{\boldsymbol{f}}\right|_{x_{k \mid k-1}} \tilde{\boldsymbol{v}}_{k \mid k-1}^{T} N_{k \mid k-1}^{x v} d\left[\begin{array}{c}
\boldsymbol{x}_{k} \\
\boldsymbol{v}_{k}
\end{array}\right]+\Gamma_{k} \boldsymbol{P}_{k \mid k-1}^{\omega y}
\end{aligned}
$$

Theorem 2: For system (1)-(3) and Assumption 1, the Gaussian recursive filter is given by

$$
\begin{gathered}
\boldsymbol{x}_{k+1 \mid k+1}=\boldsymbol{x}_{k+1 \mid k}+\boldsymbol{K}_{k+1 \mid k+1} \boldsymbol{\varepsilon}_{k+1} \\
\boldsymbol{P}_{k+1 \mid k+1}^{x x}=\boldsymbol{P}_{k+1 \mid k}^{x x}-\boldsymbol{K}_{k+1 \mid k+1} \boldsymbol{Q}_{k+1}^{\varepsilon} \boldsymbol{K}_{k+1 \mid k+1}^{T} \\
\boldsymbol{K}_{k+1 \mid k+1}=\boldsymbol{P}_{k+1 \mid k}^{x y}\left(\boldsymbol{Q}_{k+1}^{\varepsilon}\right)^{-1}
\end{gathered}
$$

where,

$$
\begin{aligned}
& \boldsymbol{P}_{k+1 \mid k}^{x y}= \\
& \alpha_{k+1}\left(\int \tilde{\boldsymbol{x}}_{k+1 \mid k}\left(\left.\tilde{\boldsymbol{h}}\right|_{x_{k+1 \mid k}}\right)^{T} N_{k+1 \mid k}^{x} d \boldsymbol{x}_{k+1}+\int \boldsymbol{K}_{k+1 \mid k} \tilde{\boldsymbol{v}}_{k+1 \mid k}^{T} N_{k+1 \mid k}^{x v} d\left[\begin{array}{c}
\boldsymbol{x}_{k+1} \\
\boldsymbol{v}_{k+1}
\end{array}\right]\right) \\
& \boldsymbol{\varepsilon}_{k+1}, \boldsymbol{Q}_{k+1}^{\varepsilon} N_{k+1 \mid k}^{x} \text { and } N_{k+1 \mid k}^{x v} \text { can be seen in Lemma } 1 .
\end{aligned}
$$

\section{Proof:}

From the projection theorem, we have

where

$$
\boldsymbol{x}_{k+1 \mid k+1}=\boldsymbol{x}_{k+1 \mid k}+\boldsymbol{K}_{k+1 \mid k+1} \varepsilon_{k+1}
$$

$$
\boldsymbol{K}_{k+1 \mid k+1}=\boldsymbol{P}_{k+1 \mid k}^{x y}\left(\boldsymbol{Q}_{k+1}^{\varepsilon}\right)^{-1}
$$

From (57), we have

$$
\boldsymbol{P}_{k+1 \mid k}^{x y}=E\left[\tilde{\boldsymbol{x}}_{k+1 \mid k} \boldsymbol{\varepsilon}_{k+1}^{T}\right]=\boldsymbol{K}_{k+1 \mid k+1} \boldsymbol{Q}_{k+1}^{\varepsilon}
$$

Substituting (56) and (58) into the covariance $\boldsymbol{P}_{k+1 \mid k+1}^{x x}$, we have

$$
\begin{aligned}
\boldsymbol{P}_{k+1 \mid k+1}^{x x}=E\left[\tilde{\boldsymbol{x}}_{k+1 \mid k+1} \tilde{\boldsymbol{x}}_{k+1 \mid k+1}^{T} \mid \boldsymbol{Y}_{k+1}\right] \\
=E\left[\tilde{\boldsymbol{x}}_{k+1 \mid k} \tilde{\boldsymbol{x}}_{k+1 \mid k}^{T} \mid \boldsymbol{Y}_{k+1}\right]-E\left[\tilde{\boldsymbol{x}}_{k+1 \mid k}\left(\boldsymbol{K}_{k+1 \mid k+1} \boldsymbol{\varepsilon}_{k+1}\right)^{T} \mid \boldsymbol{Y}_{k+1}\right] \\
-E\left[\left(\boldsymbol{K}_{k+1 \mid k+1} \boldsymbol{\varepsilon}_{k+1}\right) \tilde{\boldsymbol{x}}_{k+1 \mid k}^{T} \mid \boldsymbol{Y}_{k+1}\right] \\
+E\left[\boldsymbol{K}_{k+1 \mid k+1} \boldsymbol{\varepsilon}_{k+1}\left(\boldsymbol{K}_{k+1 \mid k+1} \boldsymbol{\varepsilon}_{k+1}\right)^{T} \mid \boldsymbol{Y}_{k+1}\right] \\
=\boldsymbol{P}_{k+1 \mid k}^{x x}-E\left[\tilde{\boldsymbol{x}}_{k+1 \mid k} \boldsymbol{\varepsilon}_{k+1}^{T} \mid \boldsymbol{Y}_{k+1}\right] \boldsymbol{K}_{k+1 \mid k+1}^{T} \\
-\boldsymbol{K}_{k+1 \mid k+1} E\left[\boldsymbol{\varepsilon}_{k+1} \tilde{\boldsymbol{x}}_{k+1 \mid k}^{T} \mid \boldsymbol{Y}_{k+1}\right]+\boldsymbol{K}_{k+1 \mid k+1} \boldsymbol{Q}_{k+1}^{\varepsilon} \boldsymbol{K}_{k+1 \mid k+1}^{T} \\
=\boldsymbol{P}_{k+1 \mid k}^{x x}-\boldsymbol{K}_{k+1 \mid k+1} \boldsymbol{Q}_{k+1}^{\varepsilon} \boldsymbol{K}_{k+1 \mid k+1}^{T}
\end{aligned}
$$

In (55),

$$
\begin{aligned}
& \boldsymbol{P}_{k+1 \mid k}^{x y}=E\left[\tilde{\boldsymbol{x}}_{k+1 \mid k} \tilde{\boldsymbol{y}}_{k+1 \mid k}^{T} \mid \boldsymbol{Y}_{k}\right] \\
& =\alpha_{k+1} E\left[\tilde{\boldsymbol{x}}_{k+1 \mid k} \tilde{\boldsymbol{z}}_{k+1 \mid k}^{T} \mid \boldsymbol{Y}_{k}\right] \\
& =\alpha_{k+1} \int \tilde{\boldsymbol{x}}_{k+1 \mid k}\left(\tilde{\boldsymbol{h}}_{x_{k+1 \mid k}}\right)^{T} N_{k+1 \mid k}^{x} d \boldsymbol{x}_{k+1} \\
& \quad+\alpha_{k+1} \int \tilde{\boldsymbol{x}}_{k+1 \mid k} \tilde{\boldsymbol{v}}_{k+1 \mid k}^{T} N_{k+1 \mid k}^{x v} d\left[\begin{array}{c}
\boldsymbol{x}_{k+1} \\
\boldsymbol{v}_{k+1}
\end{array}\right]
\end{aligned}
$$

Recursive computation of proposed algorithm:

1. Initialization $(k=0)$ : Initial values are $\boldsymbol{x}_{0}$ and $\boldsymbol{P}_{0}$; The number of algorithm runs is set to $M ; k=1$. While $\mathrm{k}<\mathrm{M}$

2. Let $\quad N_{0}=\min (N, k), \quad \omega_{k \mid k-N_{0}}=\omega_{0}, \quad \boldsymbol{P}_{k \mid k-N_{0}}^{\omega \omega}=\boldsymbol{Q}_{0}$, $\boldsymbol{v}_{k \mid k-N_{0}}=\boldsymbol{v}_{0}$ and $\boldsymbol{P}_{k \mid k-N_{0}}^{v v}=\boldsymbol{R}_{0}$.

Prediction:

3. (10), (11), and (12) are used to calculate $\omega_{k \mid k-1}, \boldsymbol{P}_{k \mid k-1}^{\omega \omega}$ and $\boldsymbol{K}_{k \mid k-1}^{\omega}$. By using (13) and (14), $\boldsymbol{K}_{k \mid k-1}^{\omega}$ can be computed by $\boldsymbol{K}_{k \mid k-N_{0}}^{\omega} \rightarrow \boldsymbol{K}_{k \mid k-N_{0}+1}^{\omega} \rightarrow \cdots \rightarrow \boldsymbol{K}_{k \mid k-1}^{\omega}$.

4. Compute $\boldsymbol{x}_{k+1 \mid k}, \boldsymbol{P}_{k+1 \mid k}^{x x}$, and $\boldsymbol{K}_{k+1 \mid k}$ through (45), (46), and (47).

Correction:

5. Using (5) and (6) to calculate $\boldsymbol{\varepsilon}_{k+1}$ and $\boldsymbol{Q}_{k+1}^{\varepsilon}$.

6. (28), (29), and (30) are used to calculate $v_{k+1 \mid k}, \boldsymbol{P}_{k+1 \mid k}^{v v}$, and $\boldsymbol{K}_{k+1 \mid k}^{v}$. By using (31) and (32), $\boldsymbol{K}_{k+1 \mid k}^{v}$ can be obtained by $\boldsymbol{K}_{k+1 \mid k-N_{0}+1}^{v} \rightarrow \boldsymbol{K}_{k+1 \mid k-N_{0}+2}^{v} \rightarrow \cdots \rightarrow \boldsymbol{K}_{k+1 \mid k}^{v}$.

7. Using (54), (55), and (56) compute $\boldsymbol{x}_{k+1 \mid k+1}, \boldsymbol{P}_{k+1 \mid k+1}^{x x}$ and $\boldsymbol{K}_{k+1 \mid k+1}$.

end while.

\section{NUMERICAL IMPLEMENTATION}

The CKF [7] is omitted here. $\varsigma_{i}, \zeta_{i}$, and $\xi_{i}$ represent sigma points set with suitable dimensions.

Assuming that values before the $k$ epoch have been known, details of the numerical implementation are demonstrated as follows.

\section{Numerical implementation of Lemma 2 :}

At first, rewrite (10) and (11) as follows

$$
\begin{gathered}
\omega_{k \mid k-n}=\omega_{k \mid k-n-1}+\boldsymbol{K}_{k \mid k-n}^{\omega} \varepsilon_{k-n} \\
\boldsymbol{P}_{k \mid k-n}^{\omega \omega}=\boldsymbol{P}_{k \mid k-n-1}^{\omega \omega}-\boldsymbol{K}_{k \mid k-n}^{\omega} \boldsymbol{Q}_{k-n}^{\varepsilon}\left(\boldsymbol{K}_{k \mid k-n}^{\omega}\right)^{T}
\end{gathered}
$$

Below, assuming $\omega_{k \mid k-2}$ and $\boldsymbol{P}_{k \mid k-2}^{\omega \omega}$ have been known, we calculate $\omega_{k \mid k-1}$ and $\boldsymbol{P}_{k \mid k-1}^{\omega \omega}$. 
We define

$$
\widehat{\Gamma}_{1}=\left[\begin{array}{c}
\omega_{k \mid k-3} \\
\boldsymbol{x}_{k-2 \mid k-3}
\end{array}\right], \Xi_{1}=\left[\begin{array}{cc}
\boldsymbol{P}_{k \mid k-3}^{\omega \omega} & \boldsymbol{P}_{k, k-2 \mid k-3}^{\omega x} \\
\left(\boldsymbol{P}_{k, k-2 \mid k-3}^{\omega x}\right)^{T} & \boldsymbol{P}_{k-2 \mid k-3}^{x x}
\end{array}\right]
$$

1) Factorize

$$
\Xi_{1}=\Sigma_{1} \Sigma_{1}^{T}
$$

2) Compute the cubature points

$$
\mathbf{X}_{i, k, k-2 \mid k-3}^{\omega x}=\left[\begin{array}{c}
\mathbf{X}_{i, k \mid k-3}^{\omega} \\
\mathbf{X}_{i, k-2 \mid k-3}^{x}
\end{array}\right]=\Sigma_{1} \varsigma_{i}+\widehat{\Gamma}_{1}, i=1,2, \cdots, 2(n+p)
$$

3) Compute the cubature points after propagation

$$
\begin{aligned}
& \boldsymbol{\mu}_{i, k-2 \mid k-3}^{x}=\boldsymbol{f}\left(\mathbf{X}_{i, k-2 \mid k-3}^{x}\right), i=1,2, \cdots, 2(n+p) \\
& \sigma_{i, k-2 \mid k-3}^{x}=\boldsymbol{h}\left(\mathbf{X}_{i, k-2 \mid k-3}^{x}\right), i=1,2, \cdots, 2(n+p)
\end{aligned}
$$

4) (14) in Lemma 2 can be expressed as

$$
\begin{aligned}
& \boldsymbol{P}_{k, k-1 \mid k-2}^{\omega x}=\frac{1}{2(n+p)} \sum_{i=1}^{2(n+p)} \boldsymbol{X}_{i, k \mid k-3}^{\omega}\left(\boldsymbol{\mu}_{i, k-2 \mid k-3}^{x}\right)^{T} \\
& -\frac{1}{2(n+p)} \sum_{i=1}^{2(n+p)} \boldsymbol{X}_{i, k \mid k-3}^{\omega} \alpha_{k-2}\left(\sigma_{i, k-2 \mid k-3}^{x}\right)^{T} \boldsymbol{K}_{k-1 \mid k-2}^{T} \\
& -\frac{1}{2(n+p)} \sum_{i=1}^{2(n+p)} \boldsymbol{X}_{i, k \mid k-3}^{\omega}\left(\frac{1}{2(n+p)} \sum_{i=1}^{2(n+p)} \mu_{i, k-2 \mid k-3}^{x}\right)^{T}+ \\
& \frac{1}{2(n+p)} \sum_{i=1}^{2(n+p)} \boldsymbol{X}_{i, k \mid k-3}^{\omega}\left(\frac{1}{2(n+p)} \boldsymbol{K}_{k-1 \mid k-2} \alpha_{k-2} \sum_{i=1}^{2(n+p)} \sigma_{i, k-2 \mid k-3}^{x}\right)^{T} \\
& +\left(\boldsymbol{Q}_{2}-\sum_{l=3}^{N} \boldsymbol{K}_{k \mid k-l}^{\omega} \boldsymbol{Q}_{k-l}^{\varepsilon}\left(\boldsymbol{K}_{k-2 \mid k-l}^{\omega}\right)^{T}\right) \Gamma_{k-2}^{T} \\
& -\alpha_{k-2}\left(\boldsymbol{S}_{2}-\sum_{l=3}^{N} \boldsymbol{K}_{k \mid k-l}^{\omega} \boldsymbol{Q}_{k-l}^{\varepsilon}\left(\boldsymbol{K}_{k-2 \mid k-l}^{v}\right)^{T}\right) \boldsymbol{K}_{k-1 \mid k-2}^{T}
\end{aligned}
$$

Substituting (69) into $N_{k, k-1 \mid k-2}^{\omega x}$, all values in $N_{k, k-1 \mid k-2}^{\omega x}$ have been known. Then, we calculate (13).

$$
\hat{\Gamma}_{2}=\left[\begin{array}{c}
\omega_{k \mid k-2} \\
\boldsymbol{x}_{k-1 \mid k-2}
\end{array}\right], \boldsymbol{\Xi}_{2}=\left[\begin{array}{cc}
\boldsymbol{P}_{k \mid k-2}^{\omega \omega} & \boldsymbol{P}_{k, k-1 \mid k-2}^{\omega x} \\
\left(\boldsymbol{P}_{k, k-1 \mid k-2}^{\omega x}\right)^{T} & \boldsymbol{P}_{k-1 \mid k-2}^{x x}
\end{array}\right]
$$

1) Factorize

$$
\Xi_{2}=\Sigma_{2} \Sigma_{2}^{T}
$$

2) Compute the cubature points

$\mathbf{X}_{i, k, k-1 \mid k-2}^{\omega x}=\left[\begin{array}{c}\mathbf{X}_{i, k \mid k-2}^{\omega} \\ \mathbf{X}_{i, k-1 \mid k-2}^{x}\end{array}\right]=\Sigma_{2} \varsigma_{i}+\widehat{\Gamma}_{2}, i=1,2, \cdots, 2(n+p)$
3) Compute the cubature points after propagation

$$
\sigma_{i, k-1 \mid k-2}^{x}=\boldsymbol{h}\left(\mathbf{X}_{i, k-1 \mid k-2}^{x}\right), i=1,2, \cdots, 2(n+p)
$$

4) (13) in Lemma 2 can be expressed as

$$
\begin{aligned}
& \boldsymbol{P}_{k, k-1 \mid k-2}^{\omega y}=\alpha_{k-1} \frac{1}{2(n+p)} \sum_{i=1}^{2(n+p)} \mathbf{X}_{i, k \mid k-2}^{\omega}\left(\sigma_{i, k-1 \mid k-2}^{x}\right)^{T} \\
& -\alpha_{k-1} \frac{1}{2(n+p)} \sum_{i=1}^{2(n+p)} \mathbf{X}_{i, k \mid k-2}^{\omega}\left(\frac{1}{2(n+p)} \sum_{i=1}^{2(n+p)} \sigma_{i, k-1 \mid k-2}^{x}\right)^{T} \\
& +\alpha_{k-1}\left(\boldsymbol{S}_{1}-\sum_{l=2}^{N} \boldsymbol{K}_{k \mid k-l}^{\omega} \boldsymbol{Q}_{k-l}^{\varepsilon}\left(\boldsymbol{K}_{k-1 \mid k-l}^{v}\right)^{T}\right)
\end{aligned}
$$

Substituting (74) into (12), we get $\boldsymbol{K}_{k \mid k-1}^{\omega}, \omega_{k \mid k-1}$, and $\boldsymbol{P}_{k \mid k-1}^{\omega \omega}$.

\section{Numerical implementation of Theorem 1:}

$N_{k \mid k-1}^{x}, \boldsymbol{\varepsilon}_{k}$, and $\boldsymbol{Q}_{k}^{\varepsilon}$ have been known at the $k$ epoch. $\omega_{k \mid k-1}, \boldsymbol{K}_{k \mid k-1}^{\omega}$, and $\boldsymbol{P}_{k \mid k-1}^{\omega \omega}$ have been calculated in Lemma 2 . $N_{k \mid k-1}^{x v}$ has been calculated in Lemma 1 at the last epoch. In $N_{k \mid k-1}^{x \omega}$, from (14), we have

$$
\begin{aligned}
& \boldsymbol{P}_{k \mid k-1}^{\omega x}=\int \omega_{k}\left(\left.\left(\tilde{\boldsymbol{f}}-\boldsymbol{K}_{k \mid k-1} \xi_{k-1} \tilde{h}\right)\right|_{x_{k-1 \mid k-2}}\right)^{T} N_{k, k-1 \mid k-2}^{\omega x} d\left[\begin{array}{c}
\omega_{k} \\
\boldsymbol{x}_{k-1}
\end{array}\right] \\
& +\left(\boldsymbol{Q}_{0}-\sum_{l=1}^{N} \boldsymbol{K}_{k \mid k-l}^{\omega} \boldsymbol{Q}_{k-l}^{\varepsilon}\left(\boldsymbol{K}_{k \mid k-l}^{\omega}\right)^{T}\right) \Gamma_{k-1}^{T} \\
& -\alpha_{k-1}\left(\boldsymbol{S}_{0}-\sum_{l=1}^{N} \boldsymbol{K}_{k \mid k-l}^{\omega} \boldsymbol{Q}_{k-l}^{\varepsilon}\left(\boldsymbol{K}_{k \mid k-l}^{v}\right)^{T}\right) \boldsymbol{K}_{k \mid k-1}^{T}
\end{aligned}
$$

From (72), we have

$$
\boldsymbol{\mu}_{i, k-1 \mid k-2}^{x}=\boldsymbol{f}\left(\mathbf{X}_{i, k-1 \mid k-2}^{x}\right), i=1,2, \cdots, 2(n+p)
$$

Then,

$$
\begin{aligned}
& \boldsymbol{P}_{k \mid k-1}^{\omega x}=\frac{1}{2(n+p)} \sum_{i=1}^{2(n+p)} \boldsymbol{X}_{i, k \mid k-2}^{\omega}\left(\mu_{i, k-1 \mid k-2}^{x}\right)^{T} \\
& -\frac{1}{2(n+p)} \sum_{i=1}^{2(n+p)} \boldsymbol{X}_{i, k \mid k-2}^{\omega}\left(\boldsymbol{K}_{k \mid k-1} \alpha_{k-1} \sigma_{i, k-1 \mid k-2}^{x}\right)^{T} \\
& -\frac{1}{2(n+p)} \sum_{i=1}^{2(n+p)} \boldsymbol{X}_{i, k \mid k-2}^{\omega}\left(\frac{1}{2(n+p)} \sum_{i=1}^{2(n+p)} \mu_{i, k-1 \mid k-2}^{x}\right)^{T} \\
& +\frac{1}{2(n+p)} \sum_{i=1}^{2(n+p)} \boldsymbol{X}_{i, k \mid k-2}^{\omega}\left(\frac{1}{2(n+p)} \sum_{i=1}^{2(n+p)} \boldsymbol{K}_{k \mid k-1} \alpha_{k-1} \sigma_{i, k-1 \mid k-2}^{x}\right)^{T} \\
& +\left(\boldsymbol{Q}_{1}-\sum_{l=2}^{N} \boldsymbol{K}_{k \mid k-l}^{\omega} \boldsymbol{Q}_{k-l}^{\varepsilon}\left(\boldsymbol{K}_{k-1 \mid k-l}^{\omega}\right)^{T}\right) \Gamma_{k-1}^{T} \\
& -\alpha_{k-1}\left(\boldsymbol{S}_{1}-\sum_{l=2}^{N} \boldsymbol{K}_{k \mid k-l}^{\omega} \boldsymbol{Q}_{k-l}^{\varepsilon}\left(\boldsymbol{K}_{k-1 \mid k-l}^{v}\right)^{T}\right) \boldsymbol{K}_{k \mid k-1}^{T}
\end{aligned}
$$


We define

$$
\begin{gathered}
\hat{\Gamma}_{3}=\left[\begin{array}{c}
\boldsymbol{x}_{k \mid k-1} \\
\omega_{k \mid k-1}
\end{array}\right], \Xi_{3}=\left[\begin{array}{cc}
\boldsymbol{P}_{k \mid k-1}^{x x} & \left(\boldsymbol{P}_{k \mid k-1}^{\omega x}\right)^{T} \\
\boldsymbol{P}_{k \mid k-1}^{\omega x} & \boldsymbol{P}_{k \mid k-1}^{\omega \omega}
\end{array}\right] \\
\hat{\Gamma}_{4}=\left[\begin{array}{l}
\boldsymbol{x}_{k \mid k-1} \\
\boldsymbol{v}_{k \mid k-1}
\end{array}\right], \Xi_{4}=\left[\begin{array}{cc}
\boldsymbol{P}_{k \mid k-1}^{x x} & \boldsymbol{P}_{k \mid k-1}^{x v} \\
\left(\boldsymbol{P}_{k \mid k-1}^{x v}\right)^{T} & \boldsymbol{P}_{k \mid k-1}^{v v}
\end{array}\right]
\end{gathered}
$$

1) Factorize

$$
\begin{gathered}
\boldsymbol{P}_{k \mid k-1}=\boldsymbol{M}_{k \mid k-1} \boldsymbol{M}_{k \mid k-1}^{T} \\
\Xi_{3}=\Sigma_{3} \Sigma_{3}^{T} \\
\Xi_{4}=\Sigma_{4} \Sigma_{4}^{T}
\end{gathered}
$$

2) Compute the cubature points

$$
\begin{gathered}
\mathbf{X}_{i, k \mid k-1}=\boldsymbol{M}_{k \mid k-1} \boldsymbol{\zeta}_{i}+\boldsymbol{x}_{k \mid k-1}, i=1, \cdots, 2 n \\
\mathbf{X}_{i, k \mid k-1}^{x \omega}=\left[\begin{array}{l}
\mathbf{X}_{i, k \mid k-1}^{x} \\
\mathbf{X}_{i, k \mid k-1}^{\omega}
\end{array}\right]=\Sigma_{3} \boldsymbol{\zeta}_{i}+\widehat{\Gamma}_{3}, i=1,2, \cdots, 2(n+p) \\
\mathbf{X}_{i, k \mid k-1}^{x v}=\left[\begin{array}{l}
\overline{\mathbf{X}}_{i, k \mid k-1}^{x} \\
\breve{\mathbf{X}}_{i, k \mid k-1}^{v}
\end{array}\right]=\Sigma_{4} \zeta_{i}+\widehat{\Gamma}_{4}, i=1,2, \cdots, 2(n+m)
\end{gathered}
$$

3) Compute the cubature points after propagation

$$
\begin{gathered}
\mu_{i, k \mid k-1}=\boldsymbol{f}\left(\mathrm{X}_{i, k \mid k-1}\right), i=1,2, \cdots, 2 n \\
\delta_{i, k \mid k-1}=\boldsymbol{h}\left(\mathrm{X}_{i, k \mid k-1}\right), i=1,2, \cdots, 2 n \\
\mu_{i, k \mid k-1}^{x}=\boldsymbol{f}\left(\mathbf{X}_{i, k \mid k-1}^{x}\right), i=1,2, \cdots, 2(n+p) \\
\delta_{i, k \mid k-1}^{x}=\boldsymbol{h}\left(\mathbf{X}_{i, k \mid k-1}^{x}\right), i=1,2, \cdots, 2(n+p) \\
\breve{\mu}_{i, k \mid k-1}^{x}=\boldsymbol{f}\left(\breve{\mathbf{X}}_{i, k \mid k-1}^{x}\right), i=1,2, \cdots, 2(n+m)
\end{gathered}
$$

4) Calculate mean and covariance in Theorem 1. According to (13), we have

$$
\begin{aligned}
& \boldsymbol{P}_{k \mid k-1}^{\omega y}=\alpha_{k}\left(\left.\int \tilde{\boldsymbol{h}}\right|_{x_{k \mid k-1}} \tilde{\boldsymbol{\omega}}_{k \mid k-1}^{T} N_{k \mid k-1}^{x \omega} d\left[\begin{array}{c}
\boldsymbol{x}_{k} \\
\boldsymbol{\omega}_{k}
\end{array}\right]\right)^{T} \\
& +\alpha_{k}\left(\boldsymbol{S}_{0}-\sum_{l=1}^{N} \boldsymbol{K}_{k \mid k-l}^{\omega} \boldsymbol{Q}_{k-l}^{\varepsilon}\left(\boldsymbol{K}_{k \mid k-l}^{v}\right)^{T}\right) \\
& =\alpha_{k}\left(\frac{1}{2(n+p)} \sum_{i=1}^{2(n+p)} \delta_{i, k \mid k-1}^{x} \boldsymbol{X}_{i, k \mid k-1}^{\omega}\right)^{T} \\
& -\alpha_{k}\left(\frac{1}{2(n+p)} \sum_{i=1}^{2(n+p)} \delta_{i, k \mid k-1}^{x}\left(\frac{1}{2(n+p)} \sum_{i=1}^{2(n+p)} \boldsymbol{X}_{i, k \mid k-1}^{\omega}\right)^{T}\right)^{T} \\
& +\alpha_{k}\left(\boldsymbol{S}_{0}-\sum_{l=1}^{N} \boldsymbol{K}_{k \mid k-l}^{\omega} \boldsymbol{Q}_{k-l}^{\varepsilon}\left(\boldsymbol{K}_{k \mid k-l}^{v}\right)^{T}\right)
\end{aligned}
$$

$$
\begin{aligned}
& \boldsymbol{K}_{k+1 \mid k}=\alpha_{k}\left(\Gamma_{k} \boldsymbol{P}_{k \mid k-1}^{\omega y}\right)\left(\boldsymbol{Q}_{k}^{\varepsilon}\right)^{-1}+\alpha_{k}\left(\frac{1}{2 n} \sum_{i=1}^{2 n} \mu_{i, k \mid k-1} \delta_{i, k \mid k-1}^{T}\right)\left(\boldsymbol{Q}_{k}^{\varepsilon}\right)^{-1} \\
& -\alpha_{k}\left(\frac{1}{2 n} \sum_{i=1}^{2 n} \mu_{i, k \mid k-1}\left(\frac{1}{2 n} \sum_{i=1}^{2 n} \delta_{i, k \mid k-1}\right)^{T}\right)\left(\boldsymbol{Q}_{k}^{\varepsilon}\right)^{-1} \\
& +\alpha_{k} \frac{1}{2(n+m)} \sum_{i=1}^{2(n+m)} \breve{\mu}_{i, k \mid k-1}^{x}\left(\breve{\mathbf{X}}_{i, k \mid k-1}^{v}\right)^{T}\left(\boldsymbol{Q}_{k}^{\varepsilon}\right)^{-1} \\
& -\alpha_{k}\left(\frac{1}{2(n+m)} \sum_{i=1}^{2(n+m)} \breve{\mu}_{i, k \mid k-1}^{x}\left(\frac{1}{2(n+m)} \sum_{i=1}^{2(n+m)} \breve{\mathbf{X}}_{i, k \mid k-1}^{v}\right)^{T}\right)\left(\boldsymbol{Q}_{k}^{\varepsilon}\right)^{-1} \\
& \boldsymbol{x}_{k+1 \mid k}=\frac{1}{2 n} \sum_{i=1}^{2 n} \boldsymbol{\mu}_{i, k \mid k-1}+\Gamma_{k} \boldsymbol{\omega}_{k \mid k-1}+\boldsymbol{K}_{k+1 \mid k} \boldsymbol{\varepsilon}_{k}
\end{aligned}
$$

$$
\begin{aligned}
& \boldsymbol{P}_{k+1 \mid k}=\frac{1}{2 n} \sum_{i=1}^{2 n} \mu_{i, k \mid k-1} \mu_{i, k \mid k-1}^{T}-\frac{1}{2 n} \sum_{i=1}^{2 n} \mu_{i, k \mid k-1}\left(\frac{1}{2 n} \sum_{i=1}^{2 n} \mu_{i, k \mid k-1}\right)^{T} \\
& +\frac{1}{2(n+p)} \sum_{i=1}^{2(n+p)} \mu_{i, k \mid k-1}^{x}\left(\mathbf{X}_{i, k \mid k-1}^{\omega}\right)^{T} \Gamma_{k}^{T} \\
& -\frac{1}{2(n+p)} \sum_{i=1}^{2(n+p)} \mu_{i, k \mid k-1}^{x}\left(\frac{1}{2(n+p)} \sum_{i=1}^{2(n+p)} \Gamma_{k} \mathbf{X}_{i, k \mid k-1}^{\omega}\right)^{T} \\
& +\left(\frac{1}{2(n+p)} \sum_{i=1}^{2(n+p)} \mu_{i, k \mid k-1}^{x}\left(\mathbf{X}_{i, k \mid k-1}^{\omega}\right)^{T} \Gamma_{k}^{T}\right. \\
& \left.-\frac{1}{2(n+p)} \sum_{i=1}^{2(n+p)} \mu_{i, k \mid k-1}^{x}\left(\frac{1}{2(n+p)} \sum_{i=1}^{2(n+p)} \Gamma_{k} \mathbf{X}_{i, k \mid k-1}^{\omega}\right)^{T}\right)^{T} \\
& +\Gamma_{k} P_{k \mid k-1}^{\omega \omega} \Gamma_{k}^{T}-\boldsymbol{K}_{k+1 \mid k} \boldsymbol{Q}_{k}^{\varepsilon} \boldsymbol{K}_{k+1 \mid k}^{T}
\end{aligned}
$$

\section{Numerical implementation of Lemma 3:}

At first, we rewrite (28) and (29) as follows

$$
\begin{gathered}
\boldsymbol{v}_{k \mid k-n}=\boldsymbol{v}_{k \mid k-n-1}+\boldsymbol{K}_{k \mid k-n}^{v} \boldsymbol{\varepsilon}_{k-n} \\
\boldsymbol{P}_{k \mid k-n}^{v v}=\boldsymbol{P}_{k \mid k-n-1}^{v v}-\boldsymbol{K}_{k \mid k-n}^{v} \boldsymbol{Q}_{k-n}^{\varepsilon}\left(\boldsymbol{K}_{k \mid k-n}^{v}\right)^{T}
\end{gathered}
$$

Below, assuming $\boldsymbol{v}_{k+1 \mid k-1}$ and $\boldsymbol{P}_{k+1 \mid k-1}^{v v}$ have been known, we calculate $\boldsymbol{v}_{k+1 \mid k}$ and $\boldsymbol{P}_{k+1 \mid k}^{v v}$.

We define

$$
\hat{\Gamma}_{5}=\left[\begin{array}{c}
\boldsymbol{v}_{k+1 \mid k-2} \\
\boldsymbol{x}_{k-1 \mid k-2}
\end{array}\right], \boldsymbol{\Xi}_{5}=\left[\begin{array}{cc}
\boldsymbol{P}_{k+1 \mid k-2}^{v v} & \boldsymbol{P}_{k+1, k-1 \mid k-2}^{v x} \\
\left(\boldsymbol{P}_{k+1, k-1 \mid k-2}^{v x}\right)^{T} & \boldsymbol{P}_{k-1 \mid k-2}^{x x}
\end{array}\right]
$$

1) Factorize

$$
\Xi_{5}=\Sigma_{5} \Sigma_{5}^{T}
$$


2) Compute the cubature points

$$
\mathbf{X}_{i, k+1, k-1 \mid k-2}^{v x}=\left[\begin{array}{l}
\mathbf{X}_{i, k+1 \mid k-2}^{v} \\
\mathbf{X}_{i, k-1 \mid k-2}^{x}
\end{array}\right]=\Sigma_{5} \varsigma_{i}+\widehat{\Gamma}_{5}, i=1, \cdots, 2(n+m)
$$

3) Compute the cubature points after propagation

$$
\begin{aligned}
& \mu_{i, k-1 \mid k-2}^{x}=\boldsymbol{f}\left(\mathbf{X}_{i, k-1 \mid k-2}^{x}\right), i=1,2, \cdots, 2(n+m) \\
& \sigma_{i, k-1 \mid k-2}^{x}=\boldsymbol{h}\left(\mathbf{X}_{i, k-1 \mid k-2}^{x}\right), i=1,2, \cdots, 2(n+m)
\end{aligned}
$$

4) (32) in Lemma 3 can be expressed as

$$
\begin{aligned}
& \boldsymbol{P}_{k+1, k \mid k-1}^{v x}=\frac{1}{2(n+m)} \sum_{i=1}^{2(n+m)} \boldsymbol{X}_{i, k+1 \mid k-2}^{v}\left(\mu_{i, k-1 \mid k-2}^{x}\right)^{T} \\
& -\frac{1}{2(n+m)} \sum_{i=1}^{2(n+m)} \boldsymbol{X}_{i, k+1 \mid k-2}^{v}\left(\boldsymbol{K}_{k \mid k-1} \alpha_{k-1} \sigma_{i, k-1 \mid k-2}^{x}\right)^{T} \\
& -\frac{1}{2(n+m)} \sum_{i=1}^{2(n+m)} \boldsymbol{X}_{i, k+1 \mid k-2}^{v}\left(\frac{1}{2(n+m)} \sum_{i=1}^{2(n+m)} \mu_{i, k-1 \mid k-2}^{x}\right)^{T} \\
& +\frac{1}{2(n+m)} \sum_{i=1}^{2(n+m)} \boldsymbol{X}_{i, k+1 \mid k-2}^{v}\left(\frac{1}{2(n+m)} \boldsymbol{K}_{k \mid k-1} \alpha_{k-1} \sum_{i=1}^{2(n+m)} \sigma_{i, k-1 \mid k-2}^{x}\right)^{T} \\
& +\left(\boldsymbol{S}_{-2}^{T}-\sum_{l=2}^{N-1} \boldsymbol{K}_{k+1 \mid k-l}^{\omega} \boldsymbol{Q}_{k-l}^{\varepsilon}\left(\boldsymbol{K}_{k-1 \mid k-l}^{\omega}\right)^{T}\right) \Gamma_{k-1}^{T} \\
& -\alpha_{k-1}\left(\boldsymbol{R}_{2}-\sum_{l=2}^{N-1} \boldsymbol{K}_{k+1 \mid k-l}^{\omega} \boldsymbol{Q}_{k-l}^{\varepsilon}\left(\boldsymbol{K}_{k-1 \mid k-l}^{v}\right)^{T}\right) \boldsymbol{K}_{k \mid k-1}^{T}
\end{aligned}
$$

Substituting (102) into $N_{k+1, k \mid k-1}^{v x}$, then, we calculate (31).

$$
\widehat{\Gamma}_{6}=\left[\begin{array}{c}
\boldsymbol{v}_{k+1 \mid k-1} \\
\boldsymbol{x}_{k \mid k-1}
\end{array}\right], \boldsymbol{\Xi}_{6}=\left[\begin{array}{cc}
\boldsymbol{P}_{k+1 \mid k-1}^{v v} & \boldsymbol{P}_{k+1, k \mid k-1}^{v x} \\
\left(\boldsymbol{P}_{k+1, k \mid k-1}^{v x}\right)^{T} & \boldsymbol{P}_{k \mid k-1}^{x x}
\end{array}\right]
$$

1) Factorize

$$
\Xi_{6}=\Sigma_{6} \Sigma_{6}^{T}
$$

2) Compute the cubature points

$$
\mathbf{X}_{i, k+1, k \mid k-1}^{v x}=\left[\begin{array}{c}
\mathbf{X}_{i, k+1 \mid k-1}^{v} \\
\mathbf{X}_{i, k \mid k-1}^{x}
\end{array}\right]=\Sigma_{6} \varsigma_{i}+\widehat{\Gamma}_{6}, i=1,2, \cdots, 2(n+m)(
$$

3) Compute the cubature points after propagation

$$
\sigma_{i, k \mid k-1}^{x}=\boldsymbol{h}\left(\mathrm{X}_{i, k \mid k-1}^{x}\right), i=1,2, \cdots, 2(n+m)
$$

4) (31) in Lemma 2 can be expressed as

$$
\begin{aligned}
& \boldsymbol{P}_{k+1, k \mid k-1}^{v y}=\alpha_{k} \frac{1}{2(n+m)} \sum_{i=1}^{2(n+m)} \mathbf{X}_{i, k+1 \mid k-1}^{v}\left(\sigma_{i, k \mid k-1}^{x}\right)^{T} \\
& -\alpha_{k} \frac{1}{2(n+m)} \sum_{i=1}^{2(n+m)} \mathbf{X}_{i, k+1 \mid k-1}^{v}\left(\frac{1}{2(n+m)} \sum_{i=1}^{2(n+m)} \sigma_{i, k \mid k-1}^{x}\right)^{T} \\
& +\alpha_{k}\left(\boldsymbol{R}_{1}-\sum_{l=1}^{N-1} \boldsymbol{K}_{k+1 \mid k-l}^{v} \boldsymbol{Q}_{k-l}^{\varepsilon}\left(\boldsymbol{K}_{k \mid k-l}^{v}\right)^{T}\right)
\end{aligned}
$$

Substituting (107) into (30), we get $\boldsymbol{K}_{k+1 \mid k}^{v}, \boldsymbol{v}_{k+1 \mid k}$, and $\boldsymbol{P}_{k+1 \mid k}^{v v}$.

\section{Numerical implementation of Lemma 1:}

To calculate $\boldsymbol{P}_{k+1 \mid k}^{x v}$ in $N_{k+1 \mid k}^{x v}$, from (105), we have

$$
\mu_{i, k \mid k-1}^{x}=\boldsymbol{f}\left(\mathbf{X}_{i, k \mid k-1}^{x}\right), i=1,2, \cdots, 2(n+m)
$$

According to (42) in Lemma 3, we have

$$
\begin{aligned}
& \boldsymbol{P}_{k+1 \mid k}^{x v}=\left(\boldsymbol{P}_{k+1 \mid k}^{v x}\right)^{T} \\
& =\left(\begin{array}{l}
\int \boldsymbol{v}_{k+1}\left(\left.\left(\tilde{\boldsymbol{f}}-\boldsymbol{K}_{k+1 \mid k} \alpha_{k} \tilde{\boldsymbol{h}}\right)\right|_{x_{k \mid k-1}}\right)^{T} N_{k+1, k \mid k-1}^{v x} d\left[\begin{array}{c}
\boldsymbol{v}_{k+1} \\
x_{k}
\end{array}\right] \\
+\boldsymbol{P}_{k+1, k \mid k-1}^{v \omega} \Gamma_{k-n}^{T}-\alpha_{k} \boldsymbol{P}_{k+1, k \mid k-1}^{v v} \boldsymbol{K}_{k+1 \mid k}^{T}
\end{array}\right)^{T} \\
& =\frac{1}{2(n+m)} \sum_{i=1}^{2(n+m)} \boldsymbol{\mu}_{i, k \mid k-1}^{x}\left(\boldsymbol{X}_{i, k+1 \mid k-1}^{v}\right)^{T} \\
& -\frac{1}{2(n+m)} \sum_{i=1}^{2(n+m)} \boldsymbol{K}_{k+1 \mid k} \alpha_{k} \boldsymbol{\sigma}_{i, k \mid k-1}^{x}\left(\boldsymbol{X}_{i, k+1 \mid k-1}^{v}\right)^{T} \\
& -\frac{1}{2(n+m)} \sum_{i=1}^{2(n+m)} \boldsymbol{\mu}_{i, k \mid k-1}^{x}\left(\frac{1}{2(n+m)} \sum_{i=1}^{2(n+m)} \boldsymbol{X}_{i, k+1 \mid k-1}^{v}\right)^{T} \\
& +\frac{1}{2(n+m)} \boldsymbol{K}_{k+1 \mid k} \alpha_{k} \sum_{i=1}^{2(n+m)} \sigma_{i, k \mid k-1}^{x}\left(\frac{1}{2(n+m)} \sum_{i=1}^{2(n+m)} \boldsymbol{X}_{i, k+1 \mid k-1}^{\nu}\right)^{T} \\
& +\left(\left(\boldsymbol{S}_{-1}^{T}-\sum_{l=1}^{N-1} \boldsymbol{K}_{k+1 \mid k-l}^{v} \boldsymbol{Q}_{k-l}^{\varepsilon}\left(\boldsymbol{K}_{k \mid k-l}^{\omega}\right)^{T}\right) \boldsymbol{\Gamma}_{k-n}^{T}\right)^{T} \\
& -\left(\alpha_{k}\left(\boldsymbol{R}_{1}-\sum_{l=1}^{N-1} \boldsymbol{K}_{k+1 \mid k-l}^{v} \boldsymbol{Q}_{k-l}^{\varepsilon}\left(\boldsymbol{K}_{k \mid k-l}^{v}\right)^{T}\right) \boldsymbol{K}_{k+1 \mid k}^{T}\right)^{T}
\end{aligned}
$$

Then, we define

$$
\widehat{\Gamma}_{7}=\left[\begin{array}{c}
\boldsymbol{x}_{k+1 \mid k} \\
\boldsymbol{v}_{k+1 \mid k}
\end{array}\right], \Xi_{7}=\left[\begin{array}{cc}
\boldsymbol{P}_{k+1 \mid k}^{x x} & \boldsymbol{P}_{k+1 \mid k}^{x v} \\
\left(\boldsymbol{P}_{k+1 \mid k}^{x v}\right)^{T} & \boldsymbol{P}_{k+1 \mid k}^{v v}
\end{array}\right]
$$

1) Factorize

$$
\Xi_{7}=\Sigma_{7} \Sigma_{7}^{T}
$$




$$
\boldsymbol{P}_{k+1 \mid k}=\boldsymbol{M}_{k+1 \mid k} \boldsymbol{M}_{k+1 \mid k}^{T}
$$

2) Compute the cubature points

$$
\begin{gathered}
\mathbf{X}_{i, k+1 \mid k}^{x v}=\left[\begin{array}{l}
\mathbf{X}_{i, k+1 \mid k}^{x} \\
\mathbf{X}_{i, k+1 \mid k}^{v}
\end{array}\right]=\Sigma_{7} \varsigma_{i}+\widehat{\Gamma}_{7}, i=1,2, \cdots, 2(n+m) \\
\mathbf{X}_{i, k+1 \mid k}=\boldsymbol{M}_{k+1 \mid k} \zeta_{i}+\boldsymbol{x}_{k+1 \mid k}, i=1, \cdots, 2 n
\end{gathered}
$$

3) Compute the cubature points after propagation

$$
\begin{gathered}
\sigma_{i, k+1 \mid k}^{x}=\boldsymbol{h}\left(\mathrm{X}_{i, k+1 \mid k}^{x}\right), i=1,2, \cdots, 2(n+m) \\
\sigma_{i, k+1 \mid k}=\boldsymbol{h}\left(\mathrm{X}_{i, k+1 \mid k}\right), i=1,2, \cdots, 2 n
\end{gathered}
$$

4) The innovation and covariance can be computed as

$$
\begin{aligned}
& \varepsilon_{k+1}=\boldsymbol{y}_{k+1}-\frac{1}{2 n} \sum_{i=1}^{2 n} \sigma_{i, k+1 \mid k}-\boldsymbol{v}_{k+1 \mid k} \\
& \boldsymbol{Q}_{k+1}^{\varepsilon}=\alpha_{k+1} \frac{1}{2 n} \sum_{i=1}^{2 n} \sigma_{i, k+1 \mid k} \sigma_{i, k+1 \mid k}^{T} \\
& -\alpha_{k+1} \frac{1}{2 n} \sum_{i=1}^{2 n} \sigma_{i, k+1 \mid k}\left(\frac{1}{2 n} \sum_{i=1}^{2 n} \sigma_{i, k+1 \mid k}\right)^{T}+\alpha_{k+1} \boldsymbol{P}_{k+1 \mid k}^{v v} \\
& +\alpha_{k+1} \frac{1}{2(n+m)} \sum_{i=1}^{2(n+m)} \sigma_{i, k+1 \mid k}^{x}\left(\mathbf{X}_{i, k+1 \mid k}^{v}\right)^{T} \\
& -\alpha_{k+1} \frac{1}{2(n+m)} \sum_{i=1}^{2(n+m)} \sigma_{i, k+1 \mid k}^{x}\left(\frac{1}{2(n+m)} \sum_{i=1}^{2(n+m)} \mathbf{X}_{i, k+1 \mid k}^{v}\right)^{T} \\
& +\alpha_{k+1}\left(\frac{1}{2(n+m)} \sum_{i=1}^{2(n+m)} \sigma_{i, k+1 \mid k}^{x}\left(\mathbf{X}_{i, k+1 \mid k}^{v}\right)^{T}\right)^{T} \\
& -\alpha_{k+1}\left(\frac{1}{2(n+m)} \sum_{i=1}^{2(n+m)} \sigma_{i, k+1 \mid k}^{x}\left(\frac{1}{2(n+m)} \sum_{i=1}^{2(n+m)} \mathbf{X}_{i, k+1 \mid k}^{v}\right)^{T}\right)^{T}
\end{aligned}
$$

\section{Numerical implementation of Theorem 2:}

From Lemma 1, we have

$$
\begin{aligned}
& \boldsymbol{P}_{k+1 \mid k}^{x y}=\alpha_{k+1} \frac{1}{2 n} \sum_{i=1}^{2 n} \mathbf{X}_{i, k+1 \mid k} \sigma_{i, k+1 \mid k}^{T} \\
& -\alpha_{k+1} \frac{1}{2 n} \sum_{i=1}^{2 n} \mathbf{X}_{i, k+1 \mid k}\left(\frac{1}{2 n} \sum_{i=1}^{2 n} \sigma_{i, k+1 \mid k}\right)^{T} \\
& +\alpha_{k+1} \frac{1}{2(n+m)} \sum_{i=1}^{2(n+m)} \mathbf{X}_{i, k+1 \mid k}^{x}\left(\mathbf{X}_{i, k+1 \mid k}^{v}\right)^{T} \\
& -\alpha_{k+1} \frac{1}{2(n+m)} \sum_{i=1}^{2(n+m)} \mathbf{X}_{i, k+1 \mid k}^{x}\left(\alpha_{k+1} \frac{1}{2(n+m)} \sum_{i=1}^{2(n+m)} \mathbf{X}_{i, k+1 \mid k}^{v}\right)^{T}
\end{aligned}
$$

From Theorem 1, Lemma 1, and Lemma 3, and substituting (119) into (56), we get $\boldsymbol{K}_{k+1 \mid k+1}, \boldsymbol{x}_{k+1 \mid k+1}$, and $\boldsymbol{P}_{k+1 \mid k+1}$.

5. Simulation

The strong nonlinearity model [11] is as follows.

$$
\boldsymbol{x}_{k+1}=\left[\begin{array}{c}
x_{1, k+1} \\
x_{2, k+1} \\
x_{3, k+1}
\end{array}\right]=\left[\begin{array}{c}
3 \sin ^{2}\left(5 x_{2, k}\right) \\
x_{1, k}+e^{-0.05 x_{3, k}}+10 \\
0.2 x_{1, k}\left(x_{2, k}+x_{3, k}\right)
\end{array}\right]+\left[\begin{array}{l}
0.1 \\
0.1 \\
0.1
\end{array}\right] \omega_{k}
$$

$$
\begin{gathered}
z_{k}=\cos \left(x_{1, k}\right)+x_{2, k} x_{3, k}+v_{k} \\
y_{k}=\gamma_{k} z_{k}+\left(1-\gamma_{k}\right) z_{k \mid k-1} \\
\omega_{k}=a_{0} \eta_{k}+a_{1} \eta_{k-1}+a_{2} \eta_{k-2}
\end{gathered}
$$

$$
v_{k}=b_{0} \eta_{k}+b_{1} \eta_{k-1}+b_{2} \eta_{k-2}
$$

The variable $\eta_{k}$ is zero-mean Gaussian white noise with variance 1. $\gamma_{k}$ is random signal with Bernoulli distribution, and the probability $p\left(\gamma_{k}=1\right)=\alpha=0.9 . a_{0}, a_{1}, a_{2}, b_{0}, b_{1}$, $b_{2}$ are set as $0.9,0.6,0.4,0.5,0.6,0.7$, respectively. The initial system state and filter state are set as $x_{0}=\left[\begin{array}{lll}-0.7 & 1 & 1\end{array}\right]^{T}, \bar{x}_{0}=\left[\begin{array}{lll}-0.7 & 1 & 1\end{array}\right]^{T}$, and $P_{0}=I_{3 \times 3}$. The Error and RMSE defined in [16] are rewritten as follows

$$
\begin{gathered}
\operatorname{Error}_{k}^{i}=\frac{1}{N} \sum_{s=1}^{N}\left(x_{i, k}^{(s)}-\hat{x}_{i, k}^{(s)}\right), 1 \leq k \leq 50, i=1, \cdots, n \\
\operatorname{RMSE}_{k}^{i}=\sqrt{\frac{1}{N} \sum_{s=1}^{N}\left(x_{i, k}^{(s)}-\hat{x}_{i, k}^{(s)}\right)^{2}}, 1 \leq k \leq 50, i=1, \cdots, n
\end{gathered}
$$

where $x_{i, k}^{(s)}$ and $\hat{x}_{i, k}^{(s)}$ represent the original state and estimated values at sth Monte Carlo run. Simulations are carried by 50 Monte Carlo runs.

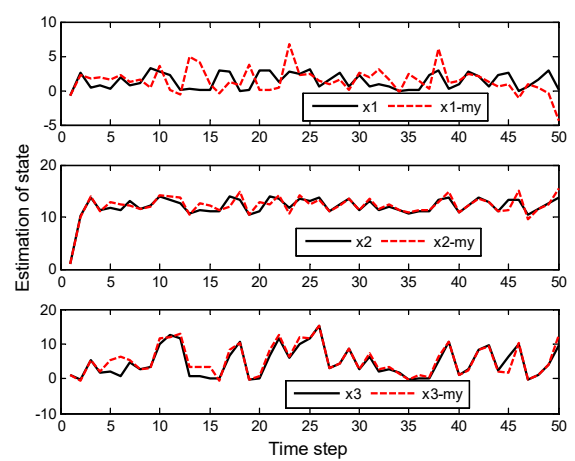

Fig.1. The estimation of the state. 

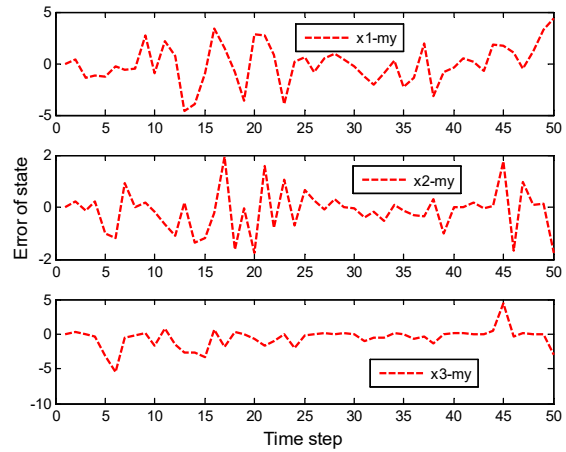

Fig.2. The Error.
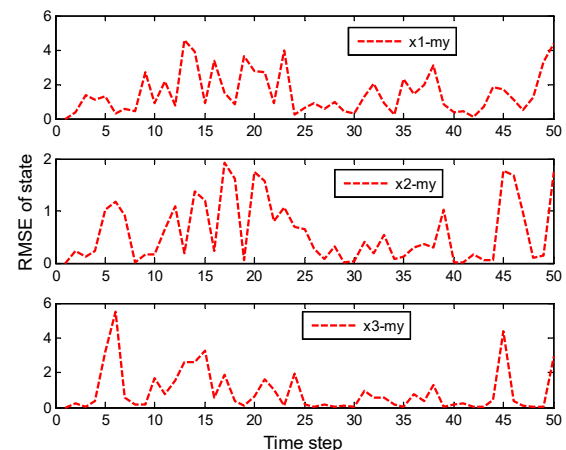

Fig.3. The RMSE.
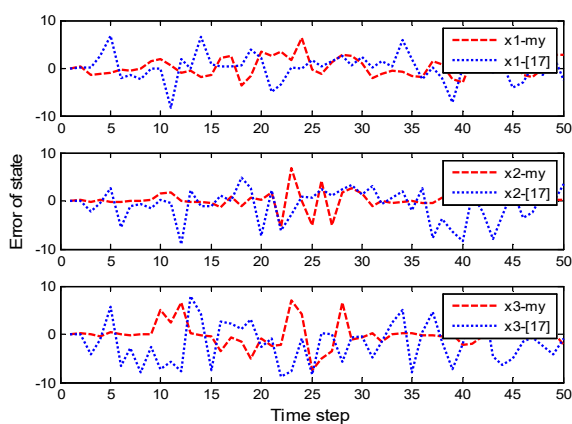

Fig.4. Comparison of the Error.
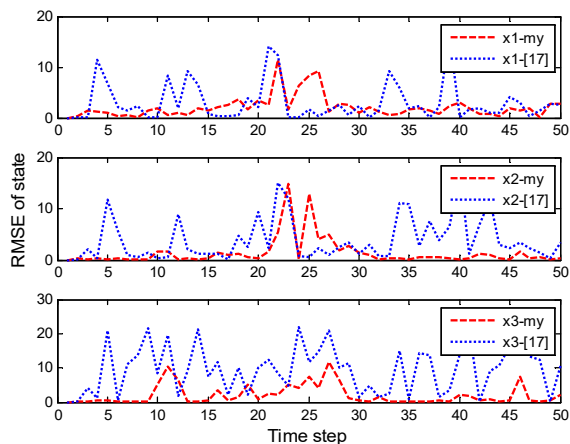

Fig.5. Comparison of the RMSE.
From Fig.1. to Fig.3., we can conclude that the derived algorithm can form a valid estimate. In Fig.1. to Fig.3., the estimated effect of the first state may be not very good, which is determined by the system, and it is consistent with the results of [10]. Fig.4.-Fig.5. are the comparison of the derived algorithm and the algorithm in [16] generalized based on EKF. Obviously, the derived algorithm can obtain higher estimation accuracy, which shows that it is necessary to design the nonlinear filtering algorithm for nonlinear systems directly compared to extending the conclusions of linear systems to nonlinear systems based on EKF.

\section{CONCLUSION}

In this paper, considering finite-step correlated noises and packet loss, a nonlinear Gaussian filtering algorithm is derived, and subsequently, corresponding numerical realization is also given. By using a strong nonlinearity model, we verified the validity of the proposed algorithm.

\section{REFERENCES}

[1] Zhao, Y.B., Kang, Y., Liu, G.P., Rees, D. (2011). Stochastic stabilization of packet-based networked control systems. International Journal of Innovative Computing, Information and Control, 7 (5), 2441-2455.

[2] Chen, G., Xia, J., Zhuang, G., Zhao, J. (2018). Improved delay-dependent stabilization for a class of networked control systems with nonlinear perturbations and two delay components. Applied Mathematics and Computation, 316, 1-17.

[3] Zhang, H., Shi, Y., Wang, J., Chen, H. (2018). A new delay-compensation scheme for networked control systems in controller area networks. IEEE Transactions on Industrial Electronics, 65 (9), 7239-7247.

[4] Bar-Shalom, Y., Li, X.R., Kirubarajan, T. (2004). Estimation with Applications to Tracking and Navigation: Theory Algorithms and Software. John Wiley \& Sons.

[5] Nørgaard, M., Poulsen, N.K., Ravn, O. (2000). New developments in state estimation for nonlinear systems. Automatica, 36 (11), 1627-1638.

[6] Ito, K. (2000). Gaussian filter for nonlinear filtering problems. In 39th IEEE Conference on Decision and Control. IEEE, 2, 1218-1223.

[7] Arasaratnam, I., Haykin, S. (2009). Cubature Kalman filters. IEEE Transactions on Automatic Control, 54 (6), 1254-1269.

[8] Doucet, A., Godsill, S., Andrieu, C. (2000). On sequential Monte Carlo sampling methods for Bayesian filtering. Statistics and Computing, 10, 197-208.

[9] Chui, C.K., Chen, G. (1989). Kalman filtering with real time applications. Applied Optics, 28, 1841.

[10] Wang, X., Liang, Y., Pan, Q., Yang, F. (2012). A Gaussian approximation recursive filter for nonlinear systems with correlated noises. Automatica, 48 (9), 2290-2297.

[11] Chang, G. (2014). Alternative formulation of the Kalman filter for correlated process and observation noise. IET Science, Measurement \& Technology, 8 (5), 310-318. 
[12] Huang, Y., Zhang, Y., Wang, X., Thao, L. (2015). Gaussian filter for nonlinear systems with correlated noises at the same epoch. Automatica, 60, 122-126.

[13] Yu, H., Zhang, X.J., Wang, S., Song, S.M. (2016). Alternative framework of the Gaussian filter for nonlinear systems with synchronously correlated noises. IET Science, Measurement \& Technology, 10 (4), 306315.

[14] Tian, T., Sun, S., Li, N. (2016). Multi-sensor information fusion estimators for stochastic uncertain systems with correlated noises. Information Fusion, 27, 126-137.

[15] Zhao, K., Li, P., Song, S.M. (2018). Gaussian filter for nonlinear stochastic uncertain systems with correlated noises. IEEE Sensors Journal, 18 (23), 9584-9594.

[16] Sun, S.L., Tian, T., Lin, H.L. (2016). Optimal linear estimators for systems with finite-step correlated noises and packet dropout compensations. IEEE Transactions on Signal Processing, 64 (21), 5672-5681.

[17] Tian, T., Sun, S.L., Lin, H.L. (2019). Distributed fusion filter for multi-sensor systems with finite-step correlated noises. Information Fusion, 46, 128-140.

[18] Sun, S.L., Wang, G.H. (2014). Modeling and estimation for networked systems with multiple random transmission delays and packet losses, Systems \& Control Letters, 73 (12), 6-16.

[19] Sun, S.L. (2013). Optimal linear filters for discrete-time systems with randomly delayed and lost measurements with/without time stamps. IEEE Transaction on Automatic Control, 58 (6), 1551-1556.
[20] Sun, S.L., Xie, L., Xiao, W., Soh, Y.C. (2008). Optimal linear estimation for systems with multiple packet dropouts. Automatica, 44 (5), 1333-1342.

[21] Zhang, W.A., Yu, L., Feng, G. (2011). Optimal linear estimation for networked systems with communication constraints. Automatica, 47 (9), 1992-2000.

[22] Ma, J., Sun, S.L. (2019). A general packet dropout compensation framework for optimal prior filter of networked multi-sensor systems. Information Fusion, 45, 128-137.

[23] Silva, E.I., Solis, M.A. (2013). An alternative look at the constant-gain Kalman filter for state estimation over erasure channels. IEEE Transaction on Automatic Control, 58 (12), 3259-3265.

[24] Ma, J., Sun, S.L. (2017). Linear estimators for networked systems with one-step random delay and multiple packet dropouts based on prediction compensation. IET Signal Processing, 11 (2), 197-204.

[25] Ding, J., Sun, S.L., Ma, J., Li, N. (2019). Fusion estimation for multi-sensor networked systems with packet loss compensation. Information Fusion, 45, 138149.

[26] Anderson, B.D.O., Moore, J.B. (2012). Optimal Filtering. Courier Corporation.

Received October 10, 2019

Accepted April 30, 2020 